\title{
The Lyman alpha reference sample
}

\section{Spatially resolved $\mathrm{H} \alpha$ kinematics ${ }^{\star} \star \star$}

\author{
Edmund Christian Herenz ${ }^{1}$, Pieter Gruyters ${ }^{2}$, Ivana Orlitova ${ }^{3}$, Matthew Hayes ${ }^{4}$, Göran Östlin ${ }^{4}$, John M. Cannon ${ }^{5}$, \\ Martin M. Roth ${ }^{1}$, Arjan Bik ${ }^{4}$, Stephen Pardy ${ }^{6}$, Héctor Otí-Floranes ${ }^{7}$, J. Miguel Mas-Hesse $^{8}$, Angela Adamo ${ }^{4}$, \\ Hakim Atek ${ }^{9}$, Florent Duval ${ }^{4,10}$, Lucia Guaita ${ }^{4,11}$, Daniel Kunth ${ }^{12}$, Peter Laursen ${ }^{13}$, Jens Melinder ${ }^{4}$, \\ Johannes Puschnig ${ }^{4}$, Thøger E. Rivera-Thorsen ${ }^{4}$, Daniel Schaerer ${ }^{14,15}$, and Anne Verhamme ${ }^{14}$ \\ ${ }^{1}$ Leibniz-Institut far Astrophysik Potsdam (AIP), An der Sternwarte 16, 14482 Potsdam, Germany \\ e-mail: cherenz@aip.de \\ 2 Lund Observatory, Box 43, 22100 Lund, Sweden \\ 3 Astronomical Institute, Academy of Sciences of the Czech Republic, Boční II 1401, 14100 Prague, Czech Republic \\ 4 Department of Astronomy, Oskar Klein Centre for Cosmoparticle Physics, Stockholm University, AlbaNova University Centre, \\ 10691 Stockholm, Sweden \\ 5 Department of Physics \& Astronomy, Macalester College, 1600 Grand Avenue, Saint Paul, MN 55105, USA \\ ${ }^{6}$ Department of Astronomy, University of Wisconsin, 475 North Charter Street, Madison, WI 53706, USA \\ 7 CONACyT research fellow - Instituto de Radioastronomía y Astrofísica, UNAM, Campus Morelia, Michoacán, CP 58089, Mexico \\ 8 Centro de Astrobiología (CSIC-INTA), Departamento de Astrofísica, PO Box 78, 28691 Villanueva de la Cañada, Spain \\ ${ }_{9}$ Laboratoire d'Astrophysique, École Polytechnique Fédérale de Lausanne (EPFL), Observatoire, 1290 Sauverny, Switzerland \\ 10 Institute for Cosmic Ray Research, The University of Tokyo, 5-1-5 Kashiwanoha, Kashiwa, 277-8582 Chiba, Japan \\ 11 INAF-Osservatorio Astronomico di Roma, via Frascati 33, 00040 Monteporzio, Italy \\ 12 Institut d'Astrophysique de Paris, UMR 7095 CNRS \& UPMC, 98bis Bd Arago, 75014 Paris, France \\ 13 Dark Cosmology Centre, Niels Bohr Institute, University of Copenhagen, Juliane Maries Vej 30, 2100 Copenhagen, Denmark \\ 14 Observatoire de Genève, Université de Genève, 51 Ch. des Maillettes, 1290 Versoix, Switzerland \\ 15 CNRS, IRAP, 14 Avenue E. Belin, 31400 Toulouse, France
}

Received 15 September 2015 / Accepted 16 November 2015

\section{ABSTRACT}

We present integral field spectroscopic observations with the Potsdam Multi-Aperture Spectrophotometer of all 14 galaxies in the $z \sim 0.1$ Lyman Alpha Reference Sample (LARS). We produce 2D line-of-sight velocity maps and velocity dispersion maps from the Balmer $\alpha(\mathrm{H} \alpha)$ emission in our data cubes. These maps trace the spectral and spatial properties of the LARS galaxies' intrinsic Ly $\alpha$ radiation field. We show our kinematic maps that are spatially registered onto the Hubble Space Telescope H $\alpha$ and Lyman $\alpha$ (Ly $\alpha$ ) images. We can conjecture a causal connection between spatially resolved $\mathrm{H} \alpha$ kinematics and Ly $\alpha$ photometry for individual galaxies, however, no general trend can be established for the whole sample. Furthermore, we compute the intrinsic velocity dispersion $\sigma_{0}$, the shearing velocity $v_{\text {shear }}$, and the $v_{\text {shear }} / \sigma_{0}$ ratio from our kinematic maps. In general LARS galaxies are characterised by high intrinsic velocity dispersions ( $54 \mathrm{~km} \mathrm{~s}^{-1}$ median) and low shearing velocities $\left(65 \mathrm{~km} \mathrm{~s}^{-1}\right.$ median). The $v_{\text {shear }} / \sigma_{0}$ values range from 0.5 to 3.2 with an average of 1.5. It is noteworthy that five galaxies of the sample are dispersion-dominated systems with $v_{\text {shear }} / \sigma_{0}<1$, and are thus kinematically similar to turbulent star-forming galaxies seen at high redshift. When linking our kinematical statistics to the global LARS Ly $\alpha$ properties, we find that dispersion-dominated systems show higher Ly $\alpha$ equivalent widths and higher Ly $\alpha$ escape fractions than systems with $v_{\text {shear }} / \sigma_{0}>1$. Our result indicates that turbulence in actively star-forming systems is causally connected to interstellar medium conditions that favour an escape of Ly $\alpha$ radiation.

Key words. galaxies: ISM - galaxies: starburst - cosmology: observations - ultraviolet: galaxies - radiative transfer

\section{Introduction}

As already envisioned by Partridge \& Peebles (1967), the hydrogen Lyman $\alpha\left(\operatorname{Ly} \alpha, \lambda_{\mathrm{Ly} \alpha}=1215.67 \AA\right)$ line has become a prominent target in successful systematic searches for galaxies in the early Universe. Redshifted into the optical, this narrow high

* Based on observations collected at the Centro Astronómico Hispano Alemán (CAHA) at Calar Alto, operated jointly by the MaxPlanck Institut für Astronomie and the Instituto de Astrofísica de Andalucía (CSIC).

$\star \star$ The reduced data cubes (FITS files) are only available at the CDS via anonymous ftp to cdsarc.u-strasbg. fr (130.79.128.5) or via http://cdsarc.u-strasbg.fr/viz-bin/qcat?J/A+A/587/A78 equivalent width line provides enough contrast to be effectively singled out in specifically designed observational campaigns. As of yet, mostly narrowband selection techniques have been employed (e.g. Hu et al. 1998; Taniguchi et al. 2003; Malhotra \& Rhoads 2004; Shimasaku et al. 2006; Tapken et al. 2006; Gronwall et al. 2007; Ouchi et al. 2008; Grove et al. 2009; Shioya et al. 2009; Hayes et al. 2010; Ciardullo et al. 2012; Sandberg et al. 2015), but multi-object and integral-field spectroscopic techniques are now also frequently used to deliver large Lyman $\alpha$ emitter (LAE) samples (Cassata et al. 2011, 2015; Adams et al. 2011; Mallery et al. 2012; Bacon et al. 2015). Moreover, all galaxy redshift record holders in the last decade 
were spectroscopically confirmed by virtue of their Ly $\alpha$ line (Iye 2011; Ono et al. 2012; Finkelstein et al. 2013; Oesch et al. 2015; Zitrin et al. 2015) and a bright LAE at $z=6.6$ is even believed to contain a significant amount of stars made exclusively from primordial material (so called Pop-III stars; Sobral et al. 2015).

As important as $\operatorname{Ly} \alpha$ radiation is in successfully unveiling star formation processes in the early Universe, its correct interpretation appears notoriously complicated. Resonant scatterings in the interstellar and circum-galactic medium diffuse the intrinsic $\operatorname{Ly} \alpha$ radiation field in real and frequency space. These scatterings increase the path length of $\operatorname{Ly} \alpha$ photons within a galaxy and consequently $\operatorname{Ly} \alpha$ is more susceptible to being destroyed by dust (see Dijkstra 2014, for a comprehensive review covering the $\operatorname{Ly} \alpha$ radiative transfer fundamentals). Consequently, a galaxy's $\operatorname{Ly} \alpha$ observables are influenced by a large number of its physical properties. Understanding these influences is crucial to correctly interpret high- $z$ LAE samples. In other words, we have to answer the question: What differentiates an LAE from other star-forming galaxies that do not show Ly $\alpha$ in emission?

Regarding Ly $\alpha$ radiation transport in individual galaxies, a large body of theoretical work investigated analytically and numerically how within simplified geometries certain parameters (e.g. density, temperature, dust content, kinematics, and clumpiness of the interstellar medium) affect the observed Ly $\alpha$ radiation field (e.g. Neufeld 1990; Ahn et al. 2003; Dijkstra et al. 2006; Verhamme et al. 2006; Laursen et al. 2013; Gronke \& Dijkstra 2014; Duval et al. 2014). Commonly a spherical shell model is adopted. In this model, a thin shell of expanding, contracting, or static neutral gas represents the medium responsible for scattering Ly $\alpha$ photons. As a result, from scattering in the shell complex Ly $\alpha$ line morphologies arise and the expansion velocity and the neutral hydrogen column density of the shell are of pivotal importance in shaping the observable $\operatorname{Ly} \alpha$ line. By introducing deviations from pure spherical symmetry, Zheng \& Wallace (2014) and Behrens et al. (2014) show that the observed Ly $\alpha$ properties also depend on the viewing angle under which a system is observed. The Behrens et al. result is also found in large-scale cosmological simulations that were postprocessed with Ly $\alpha$ radiative transport simulations (e.g. Laursen \& Sommer-Larsen 2007; Laursen et al. 2009; Barnes et al. 2011). Recently more realistic hydrodynamic simulations of isolated galaxies have been paired with $\mathrm{Ly} \alpha$ radiation transport simulations (Verhamme et al. 2012; Behrens \& Braun 2014). These studies again underline the viewing angle dependence of the Ly $\alpha$ observables. In particular, they show that disks observed face-on are expected to exhibit higher $\operatorname{Ly} \alpha$ equivalent widths and $\operatorname{Ly} \alpha$ escape fractions than if they were observed edge-on. More importantly, these state-of-the-art simulations also emphasise the importance of small-scale interstellar medium structure that was previously not included in simple models. For example Behrens \& Braun (2014) demonstrate how supernova-blown cavities are able to produce favoured escape channels for Ly $\alpha$ photons.

Observationally, when Ly $\alpha$ is seen in emission, the spectral line profiles can be typified by their distinctive shapes. In a large number of LAE spectra the Ly $\alpha$ line often appears asymmetric, with a relatively sharp drop on the blue and a more extended wing on the red side. A significant fraction of spectra also shows characteristic double peaks, with the red peak often being stronger than the blue (e.g. Tapken et al. 2004, 2007; Yamada et al. 2012; Hong et al. 2014; Henry et al. 2015; Yang et al. 2015). Interestingly, a high percentage of double peaked and asymmetric Ly $\alpha$ profiles appear well explained by the spherical symmetric scenarios mentioned above. Double-peaked profiles are successfully reproduced by slowly-expanding shells $\left(v_{\text {exp }} \lesssim 100 \mathrm{~km} \mathrm{~s}^{-1}\right)$ or low neutral hydrogen column densities $N_{\mathrm{HI}} \lesssim 10^{19} \mathrm{~cm}^{-2}$, while high expansion velocities $\left(v_{\exp } \sim 150-\right.$ $300 \mathrm{~km} \mathrm{~s}^{-1}$ ) with neutral hydrogen column densities of $N_{\mathrm{HI}} \gtrsim$ $10^{20} \mathrm{~cm}^{-2}$ produce the characteristic asymmetric profile with an extended red wing (Tapken et al. 2006, 2007; Verhamme et al. 2008; Schaerer et al. 2011; Gronke \& Dijkstra 2014).

Further observational constraints on the kinematics of the scattering medium can be obtained by measuring the offset from non-resonant, rest-frame optical emission lines (e.g. $\mathrm{H} \alpha$ or [O III]) to low-ionisation state metal absorption lines (e.g. O I $\lambda 1302$ or Si II $\lambda 1304)$. While the emission lines provide systemic redshift, some of metal absorption lines in a low ionisation state trace the kinematics of the cold neutral gas phase. Both observables are challenging to obtain for high- $z$ LAEs and require long integration times on $8-10 \mathrm{~m}$ class telescopes (e.g. Shapley et al. 2003; Tapken et al. 2004) or even additional help from gravitational lenses (e.g. Schaerer \& Verhamme 2008; Christensen et al. 2012). As the continuum absorption often remains undetected, just the offset between the rest-frame optical lines and the Ly $\alpha$ peaks are measured (e.g. McLinden et al. 2011; Guaita et al. 2013; Erb et al. 2014). Curiously, the observed Ly $\alpha$ profiles also agree well with those predicted by the simple shell model, when the measured offsets (typically $\Delta v \sim 200 \mathrm{~km} \mathrm{~s}^{-1}$ ) are associated with shell expansion velocities in the simple shell model (Verhamme et al. 2008; Hashimoto et al. 2013; Song et al. 2014; Hashimoto et al. 2015). Only a few profiles appear incompatible with the expanding shells (Chonis et al. 2013). These profiles are characterised by extended wings or bumps in the blue side of the profile (Martin et al. 2014; Henry et al. 2015). However, given the aforementioned viewing angle dependencies in more complex scenarios, this overall success of the simple shell model appears surprising and is therefore currently under scrutiny (Gronke et al. 2015). Nevertheless, at least qualitatively the observations demonstrate, in concert with theoretical predictions, that LAEs predominantly have outflow kinematics and that such outflows promote the Ly $\alpha$ escape (see also Kunth et al. 1998; Mas-Hesse et al. 2003).

Kinematic information is moreover encoded in the restframe optical line emission alone. These emission lines trace the ionised gas kinematics and, in particular, the hydrogen recombination lines such as $\mathrm{H} \alpha$ relate directly to the spatial and spectral properties of a galaxy's intrinsic Ly $\alpha$ radiation field. Therefore spatially resolved spectroscopy of a galaxy's $\mathrm{H} \alpha$ radiation field constrains the initial conditions for the subsequent Ly $\alpha$ radiative transfer through the interstellar, circum-galactic, and intergalactic medium to the observer. At high $z$, however, most LAEs are so compact that they cannot be spatially resolved from the ground and, hence, all spatial information is lost in the analysis of the integrated spectra. Resolving the intrinsic Ly $\alpha$ radiation of typical high- $z$ LAEs spatially and spectrally at such small scales would require integral field spectroscopy, preferably with adaptive optics, in the near infrared with long integration times. Although large samples of continuum-selected $z \sim 2-3$ star-forming galaxies have already been observed with this method (see Glazebrook 2013, for a comprehensive review), little is known about the $\operatorname{Ly} \alpha$ properties of the galaxies in those samples.

In this paper we present results obtained from our integralfield spectroscopic observations with the aim to relate spatially and spectrally resolved intrinsic Ly $\alpha$ radiation field to its observed Ly $\alpha$ properties. Therefore we targeted the $\mathrm{H} \alpha$ line in all galaxies of the $z \sim 0.03-0.18$ Lyman alpha reference sample (LARS). The sample consists of 14 nearby laboratory galaxies, 
that have far-UV (FUV, $\lambda \sim 1500 \AA$ ) luminosities similar to those of high- $z$ star-forming galaxies. Moreover, to ensure a strong intrinsic Ly $\alpha$ radiation field, galaxies with large $\mathrm{H} \alpha$ equivalent widths were selected $\left(E W_{\mathrm{H} \alpha} \geq 100 \AA\right)$. The backbone of LARS is a substantial program with the Hubble Space Telescope (HST). In this program, each galaxy was observed with a combination of ultraviolet long-pass filters, optical broadband filters, as well as $\mathrm{H} \alpha$ and $\mathrm{H} \beta$ narrowband filters. These images were used to accurately reconstruct $\operatorname{Ly} \alpha$ images of those 14 galaxies. Moreover, UV spectroscopy with HSTs Cosmic Origins Spectrograph (COS) is available for the whole sample.

This paper is the seventh in a series presenting results of the LARS project. In Östlin et al. (2014; hereafter Paper I) we detailed the sample selection, the observations with HST and the process to reconstruct Ly $\alpha$ images from the HST data. In Hayes et al. (2014; hereafter Paper II) we presented a detailed analysis of the imaging results. We found that six of the 14 galaxies are indeed analogous to high- $z$ LAEs, i.e. they would be selected by the conventional narrowband survey selection requirement ${ }^{1}$ : $E W_{\mathrm{Ly} \alpha}>20 \AA$. The main result of Paper II is that a galaxy's morphology seen in $\operatorname{Ly} \alpha$ is usually very different compared to its morphological appearance in $\mathrm{H} \alpha$ and the FUV; in particular, Ly $\alpha$ is often less centrally concentrated and so these galaxies are embedded in a faint low surface brightness Ly $\alpha$ halo. The results in Paper II (see also Hayes et al. 2013) therefore provide clear observational evidence for resonant scattering of Ly $\alpha$ photons in the neutral interstellar medium. Pardy et al. (2014; hereafter Paper III) subsequently presented $21 \mathrm{~cm}$ observations tracing the neutral hydrogen content of the LARS galaxies and the results supported the complex coupling between Ly $\alpha$ radiative transfer and the properties of the neutral medium. In Guaita et al. (2015; hereafter Paper IV), the morphology of the LARS galaxies was thoroughly re-examined. By artificially redshifting the LARS imaging data-products, it was confirmed that morphologically LARS galaxies indeed resemble $z \sim 2-3$ star forming galaxies. Rivera-Thorsen et al. (2015; hereafter Paper V) then presented high-resolution FUV COS spectroscopy of the full sample. Analysing the neutral interstellar medium kinematics as traced by the low-ionisation state metal absorption lines, it was shown that all galaxies with global $\operatorname{Ly} \alpha$ escape fractions $>5 \%$ appear to have outflowing winds. Finally, in Duval et al. (2016; hereafter Paper VI), a detailed radiative transfer study of one LARS galaxy was performed using all the observational constraints assembled within the LARS project, and including data that is presented in this paper. In particular it was shown that this galaxy's spatial and spectral Ly $\alpha$ emission properties are consistent with scattering of Ly $\alpha$ photons by outflowing cool material along the minor axis of the disk.

Now, in the first analysis of our LARS integral-field spectroscopic data presented here, we focus on a comparison of results obtainable from spatially resolved $\mathrm{H} \alpha$ kinematics to results from the LARS HST Ly $\alpha$ imaging and $21 \mathrm{~cm}$ H I observations. In a subsequent publication (Orlitova, in prep.) we will combine information from our 3D $\mathrm{H} \alpha$ spectroscopy with our COS UV spectra to constrain the parameters of outflowing winds.

This paper is not the first in relating observed spatially resolved $\mathrm{H} \alpha$ observations to a local galaxy's Ly $\alpha$ radiation field. Recently in a pioneering study exploiting MUSE (Bacon et al. 2014) science verification data, Bik et al. (2015) showed that an asymmetric $\operatorname{Ly} \alpha$ halo around the main star-forming knot of

\footnotetext{
1 We adopt the convention established in high- $z$ narrowband surveys of designating galaxies with $E W_{\mathrm{Ly} \alpha} \geq 20 \AA$, LAEs, and galaxies with $E W_{\text {Ly } \alpha}<20 \AA$, non-LAEs.
}

ESO338-IG04 (Hayes et al. 2005; Östlin et al. 2009; Sandberg et al. 2013) can be linked to outflows seen in the $\mathrm{H} \alpha$ radial velocity field. Moreover, the kinematic constraints provided by our observations were already used for modelling the Ly $\alpha$ scattering in one LARS galaxy (Paper VI), and also here galactic scale outflows were required to explain the galaxy's Ly $\alpha$ radiation. With the full data set presented here, we now study whether such theoretical expected effects are indeed common among Ly $\alpha$-emitting galaxies.

The outline of this manuscript is as follows: in Sect. 2 our PMAS observations of the LARS sample are detailed. The reduction of our PMAS data is explained in Sect. 3. Ancillary LARS data products used in this manuscript are described briefly in Sect. 4. The derivation and analysis of the $\mathrm{H} \alpha$ velocity and dispersion maps is presented in Sect. 5. In Sect. 6 the results are discussed and finally we summarise and conclude in Sect. 7. Notes on individual objects are given in Appendix A.

\section{Observations with PMAS}

We observed all LARS galaxies with the Potsdam MultiAperture Spectrophotometer (PMAS; Roth et al. 2005) at the Calar Alto $3.5 \mathrm{~m}$ telescope during four nights from March 12 to March 15, 2012 (PMAS run212), except for LARS 13, which was observed on October 102011 (PMAS run197). We used PMAS in the lens array configuration, where 256 fibers are coupled to a $16 \times 16$ lens array that contiguously samples the sky. Depending on the extent of the targeted galaxy, we used either the standard magnification mode, which provides an $8^{\prime \prime} \times 8^{\prime \prime}$ field of view (FoV) or the double magnification mode ${ }^{2}$, where the FoV is $16^{\prime \prime} \times 16^{\prime \prime}$. The backwards-blazed R1200 grating was mounted on the spectrograph. To ensure proper sampling of the line spread function the $4 \mathrm{k} \times 4 \mathrm{k}$ CCD (Roth et al. 2010) was read out unbinned along the dispersion axis. This set-up delivers a nominal resolving power from $R \sim 5000$ to $R \sim 8000$ within the targeted wavelength ranges ${ }^{3}$. In Sect. 3.3 we show that while the nominal resolving powers are met on average, the instrumental broadening varies at small amplitudes from fibre to fibre.

On-target exposures were usually flanked by $400 \mathrm{~s}$ exposures of empty sky near the target. These sky frames serve as a reference for removing the telluric background emission. Owing to an error in our observing schedule, no sky frames were taken for LARS 4, LARS 7, and LARS 9 (2012-03-12 pointing). Fortunately, this did not render the observations unusable, since there are enough blank-sky spectral pixels (so called spaxels) within those on-target frames to provide us with a reference sky (cf. Sect. 3.2).

Observing blocks of one hour were usually flanked by continuum and $\mathrm{HgNe}$ arc lamp exposures used for photometric and wavelength calibration. We obtained several bias frames throughout each night when the detector was idle during target acquisition. Spectrophotometric standard stars were observed at the beginning and at the end of each astronomical night $\left(\mathrm{BD}+75 \mathrm{~d} 325 \&\right.$ Feige 67 from Oke 1990, $\left.t_{\text {exp. }}=600 \mathrm{~s}\right)$. Twilight flat exposures were taken during dawn and dusk.

\footnotetext{
2 The naming of the mode refers to the instruments internal magnification of the telescopes focal plane; doubling the magnification of the focal plane doubles the extent of the FoV.

3 Values taken from the PMAS online grating tables, available at http://www . caha.es/pmas/PMAS_COOKBOOK/TABLES/pmas_ gratings.html\#4K_1200_1BW
} 
Table 1. Log of PMAS lens array observations of the LARS sample.

\begin{tabular}{cccccccc}
\hline \hline $\begin{array}{c}\text { LARS } \\
\text { ID }\end{array}$ & $\begin{array}{c}\text { Observing- } \\
\text { date }\end{array}$ & $\begin{array}{c}\text { exp. } \\
{[\mathrm{s}]}\end{array}$ & $\begin{array}{c}\text { FoV } \\
{\left[\operatorname{arcsec}^{2}\right]}\end{array}$ & $\begin{array}{c}\lambda \text {-range } \\
\lambda_{\text {start }}-\lambda_{\text {end }}[\AA]\end{array}$ & $\begin{array}{c}\text { Resolving power } \\
\bar{R}_{\text {FWHM }}\end{array}$ & $\begin{array}{c}\text { Seeing } \\
F W H M\left[{ }^{\prime \prime}\right]\end{array}$ & $\begin{array}{c}\text { Observing- } \\
\text { conditions }\end{array}$ \\
\hline 1 & $2012-03-16$ & $2 \times 1800 \mathrm{~s}$ & $16 \times 16$ & $5948-7773$ & 5306 & 1.8 & phot. \\
2 & $2012-03-15$ & $2 \times 1800 \mathrm{~s}$ & $16 \times 16$ & $5752-7581$ & 5205 & 1.1 & non-phot. \\
3 & $2012-03-14$ & $3 \times 1800 \mathrm{~s}$ & $16 \times 16$ & $5948-7773$ & 5362 & 1.1 & phot. \\
4 & $2012-03-13$ & $3 \times 1800 \mathrm{~s}$ & $16 \times 16$ & $5752-7581$ & 5883 & 1.0 & phot. \\
5 & $2012-03-14$ & $3 \times 1800 \mathrm{~s}$ & $8 \times 8$ & $5874-7700$ & 5886 & 1.3 & phot. \\
6 & $2012-03-13$ & $2 \times 1800 \mathrm{~s}$ & $16 \times 16$ & $5752-7581$ & 5865 & 1.0 & phot. \\
7 & $2012-03-16$ & $3 \times 1800 \mathrm{~s}$ & $16 \times 16$ & $5948-7773$ & 3925 & 1.3 & non-phot. \\
8 & $2012-03-15$ & $3 \times 1800 \mathrm{~s}$ & $16 \times 16$ & $5948-7773$ & 4415 & 0.9 & non-phot. \\
9 & $2012-03-12$ & $3 \times 1800 \mathrm{~s}$ & $16 \times 16$ & $5752-7581$ & 5561 & 0.9 & phot. \\
& $2012-03-14$ & $3 \times 1800 \mathrm{~s}$ & $16 \times 16$ & $5948-7773$ & 4772 & 0.9 & non-phot. \\
10 & $2012-03-14$ & $2 \times 1800 \mathrm{~s}$ & $8 \times 8$ & $5874-7700$ & 5438 & 1.5 & phot. \\
11 & $2012-03-15$ & $3 \times 1800 \mathrm{~s}$ & $16 \times 16$ & $5948-7773$ & 4593 & 1.0 & non-phot. \\
12 & $2012-03-14$ & $3 \times 1800 \mathrm{~s}$ & $8 \times 8$ & $6553-8363$ & 6756 & 0.9 & phot. \\
13 & $2011-10-02$ & $4 \times 1800 \mathrm{~s}$ & $8 \times 8$ & $6792-8507$ & 7766 & 1.2 & phot. \\
& $2011-10-02$ & $4 \times 1800 \mathrm{~s}$ & $8 \times 8$ & $6792-8507$ & 7771 & 1.2 & phot. \\
14 & $2012-03-13$ & $2 \times 1800 \mathrm{~s}$ & $8 \times 8$ & $6553-8363$ & 7718 & 0.9 & phot. \\
\hline
\end{tabular}

Notes. See Paper I for coordinates and common names of the galaxies in the LARS sample. Galaxies LARS 09 and LARS 13 were covered with two pointings each, since their extent was larger than the used FoV.

In Table 1 we provide a log of our observations. By changing the rotation of the grating, we adjusted the wavelength ranges covered by the detector, such that the galaxies $\mathrm{H} \alpha-[\mathrm{N}$ II $]$ complex is located near the centre of the CCD. We emphasise that $\pm 350 \AA$ at the upper/lower end of the quoted spectral ranges are affected by vignetting (a known "feature" of the PMAS detector; see Roth et al. 2010). We also quote the average spectral resolution of our final data sets near $\mathrm{H} \alpha$. The determination of this quantity is described in Sect. 3.3. The tabulated seeing values refer always to the average full width at half maximum (FWHM) of the guide star point-spread function (PSF) measured during the exposures with the acquisition and guiding camera of PMAS. This value is on average $\approx 0.2^{\prime \prime}$ higher than the DIMM measurements (see also Husemann et al. 2013).

In Fig. 1 we show the wavelengths of the redshifted $\mathrm{H} \alpha$ line for all LARS galaxies overlaid on the typical night sky emission spectrum at Calar Alto from Sánchez et al. (2007a). As can be seen for two galaxies (LARS 13 and LARS 14), the $\mathrm{H} \alpha$ line signal is contaminated by telluric line emission and two other galaxies (LARS 9 and LARS 12) have their $\mathrm{H} \alpha$ line within an absorption band. This, however, has no effect on the presented analysis. For LARS 13 and LARS 14, we could optimally subtract the interfering sky lines from the science exposures using the separate sky frames. Moreover, as we use only spaxels with high signal-to-noise $\mathrm{H} \alpha$ lines in our analysis (see also Sect. 5), the high frequency changes within the telluric absorption bands do not alter the quantified features, i.e. width and peak position, of the profiles.

\section{The PMAS data reduction}

\subsection{Basic reduction with $p 3 d$}

For reducing the raw data the p3d-package ${ }^{4}$ (Sandin et al. 2010, 2012) was utilised. This pipeline covers all basic steps needed for reducing fiber-fed integral field spectroscopic data: bias subtraction, flat fielding, cosmic ray removal, tracing and extraction of the spectra, correction of differential atmospheric refraction,

\footnotetext{
4 http://p3d.sourceforge.net
}

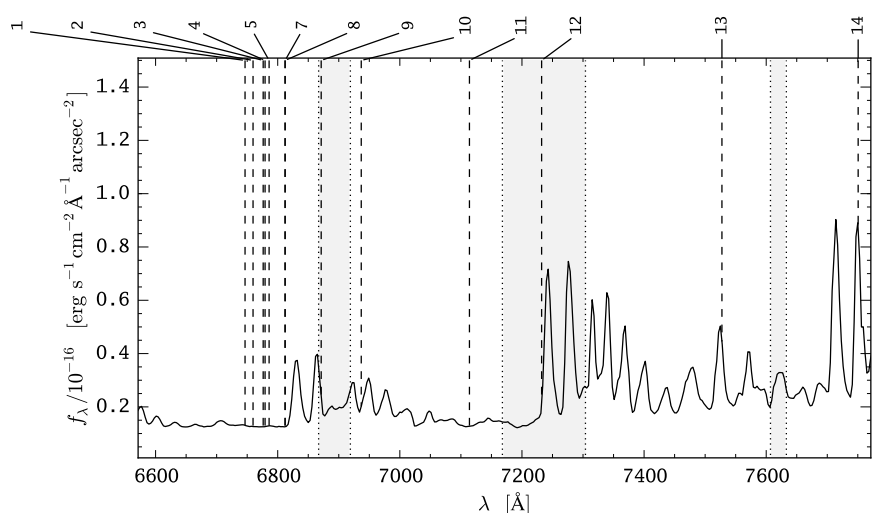

Fig. 1. Wavelengths of the redshifted $\mathrm{H} \alpha$ lines of the LARS galaxies (vertical dashed lines) compared to the night sky emission spectrum at Calar Alto (Sánchez et al. 2007a). Grey regions indicate the telluric absorption bands $\left(\mathrm{O}_{2} B\right.$-Band: $6887 \AA-6919 \AA$, $\mathrm{O}_{2} A$-Band: $7607 \AA-7633 \AA$, and $\mathrm{H}_{2} \mathrm{O} a$-Band: $7168 \AA-7304 \AA$, see e.g. Cox 2000).

and co-addition of exposures (see also Turner 2010). We now describe how we applied the tasks of p3d on our raw data for each of these steps 5 :

- Bias subtraction: Master bias frames were created by p3d_cmbias. These master bias frames were then subtracted from the corresponding science frames. Visual inspection of the bias subtracted frames showed no measurable offsets in unexposed regions between the four quadrants of the PMAS $\mathrm{CCD}$, which attests to optimal bias removal.

- Flat fielding: Each of the 256 fibers has its own wavelength dependent throughput curve. To determine these, the task p3d_cflatf was applied on the twilight flats. The determined curves are then applied in the extraction step of the science frame (see below) to normalise the extracted spectra.

5 As the amount of raw data from the observations was substantial, we greatly benefited from the scripting capabilites of $\mathrm{p} 3 \mathrm{~d}$ (Sandin et al. 2011). Our shell scripts and example parameter files can be found at https://github.com/Knusper/pmas_data_red 
- Cosmic ray removal: Cosmic ray hits on the CCD were removed with p3d_cr. Visual inspections guided by the parameter study of Husemann et al. (2012) lead us to apply the L.A. Cosmic algorithm (van Dokkum 2001) with $\sigma_{\text {lim }}=5$, $\sigma_{\text {frac }}=1, f_{\text {lim }}=15$, a grow radius of 2 and a maximum of four iterations.

- Tracing and extraction: Spectra were extracted with the modified Horne (1986) optimal extraction algorithm (Sandin et al. 2010). The task p3d_ctrace was used to determine the traces and the cross-dispersion profiles of the spectra on the detector. Spectra were then extracted with p3d_cobjex, also using the median recentring recommended in Sandin et al. (2012).

- Wavelength calibration: We obtained dispersion solutions (i.e. mappings from pixel to wavelength space) from the $\mathrm{HgNe}$ lamp frames using a sixth-order polynomial with p3d_cdmask. The wavelength sampling of the extracted science spectra is typically $0.46 \AA \mathrm{px}^{-1}$. We further improved the wavelength calibration of the science frames by applying small shifts (typically $0.1-0.3 \mathrm{px}$ ) determined from the strong $6300 \AA$ and $6364 \AA$ [O I] sky lines (not available within the targeted wavelength range for LARS 12, 13, and 14)

- Sensitivity function and flux calibration: Using extracted and wavelength-calibrated standard star spectra, we created a sensitivity function utilising p3d_sensfunc. Absorption bands in the standard star spectra, as well as telluric absorption bands (see Fig. 1), were masked for the fit. Extinction curves were created using the empirical formula presented in Sánchez et al. (2007b) with the extinction in $V$-band as measured by the Calar Alto extinction monitor ${ }^{6}$. We fluxcalibrated all extracted and wavelength-calibrated science frames with the derived sensitivity function and extinction curve with p3d_fluxcal.

The final data products from the p3d-pipeline are flux- and wavelength-calibrated data cubes for all science exposures, as well as the corresponding error cubes. From here we now perform the following reduction steps with our custom procedures written in python ${ }^{7}$.

\subsection{Sky subtraction and co-addition}

- Sky subtraction: All sky frames were reduced as science frames, as described in the previous section. These extracted, wavelength- and flux-calibrated sky frames were then subtracted from the corresponding science frames and errors were propagated accordingly. Unfortunately, no separate sky exposures were taken for three targets (LARS 4, LARS 7, and LARS 9; see also Sect. 2). For these targets we created a narrowband image of the $\mathrm{H} \alpha$-[N II] region by summing up the relevant layers in the datacube. In this image, we selected spaxels that do not contain significant amounts of flux. From these spaxels then an average sky spectrum was created, which was then subtracted from all spaxels. As the spectral resolution varies across the FoV (see also Sect. 3.3), this method produces some residuals at the position of the sky lines, which however do not touch the $\mathrm{H} \alpha$ lines of the affected targets.

- Stacking: We co-added all individual flux-calibrated and skysubtracted data cubes using the variance-weighted mean. For

\footnotetext{
6 http://www . caha.es/CAVEX/cavex.php

http://wWw . python. org
}

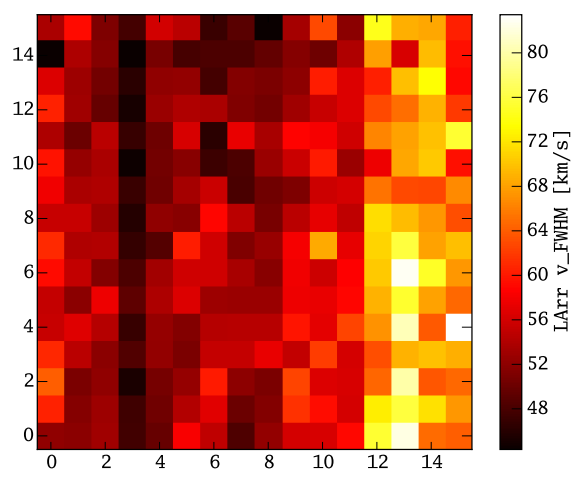

Fig. 2. Representative resolving power map for the observation of LARS 1. The resolution is expressed as $v_{\text {FWHM }}$ of a 1D Gaussian fit. Black spaxels at the positions $(x, y)=(0,14)$ and $(8,15)$ are dead fibres.

this calculation the input variances were derived from squaring the error cubes. Before co-addition we ensured by visual inspection that there are no spatial offsets between individual exposures.

\subsection{Spectral resolving power determination}

To determine the intrinsic width of our observed $\mathrm{H} \alpha$ lines, we need to correct for instrumental broadening by the spectrograph's line spread function (the spectrograph's resolving power; Robertson 2013). It is known that for PMAS the resolving power varies from fibre to fibre and with wavelength (e.g. Sánchez et al. 2012). To determine the broadening at the wavelength of the $\mathrm{H} \alpha$ line, we use the $\mathrm{HgNe}$ arc lamp exposures, which were originally used to wavelength calibrate the on-target exposures. We first create wavelength-calibrated data cubes from these arc frames. Next, we select two strong arc lamp lines that are nearest in wavelength to the corresponding galaxy's $\mathrm{H} \alpha$ line. Finally, we fit 1D Gaussians in each spaxel to each of those lines (e.g. similar to Alonso-Herrero et al. 2009). Typically the separation from a galaxy's $\mathrm{H} \alpha$ line to one of those arc lamp lines is $\sim 50 \AA$. The difference between the $F W H M$ of the fits to the different lines is typically $\sim 1 \mathrm{~km} \mathrm{~s}^{-1}$, hence we take the average of both at the position of $\mathrm{H} \alpha$ as the resolving power for each spaxel. We point out that the arc-lamp lines $F W H M$ is always sampled by more than 2 pixels, therfore aliasing effects can be neglected (Turner 2010).

This procedure provides us with resolving power maps. As an example, we show a so-derived map for the observations of LARS 1 in Fig. 2. We note that the spatial gradient seen in Fig. 2 is not universal across our observations (see Sánchez et al. 2012, for an explanation). We further note that the formal uncertainties on the resolving power determination are negligible in comparison to the uncertainties derived on the $\mathrm{H} \alpha$ profiles in Sect. 5.

In Table 1 we give the mean resolving power at the position of $\mathrm{H} \alpha$ for each galaxy as $\bar{R}_{\text {FWHM }}$. Variations within the FoV typically have an amplitude of $\sim 30 \mathrm{~km} \mathrm{~s}^{-1}$. The average resolving power of all our observations is $R=5764$ or $52 \mathrm{~km} \mathrm{~s}^{-1}$, which is consistent with the values given in the PMAS online grating manual $^{8}$.

\footnotetext{
8 http://www.caha.es/pmas/PMAS_COOKBOOK/TABLES/pmas_ gratings.html\#4K_1200_1BW
} 


\subsection{Registration on astrometric grid of HST observations}

To facilitate a comparison of our PMAS observations with the HST imaging results from Hayes et al. (2014), we have to register our PMAS data cubes with respect to the LARS HST data products. As explained in Östlin et al. (2014), all HST images are aligned with respect to each other and have a common pixel scale of $0.04^{\prime \prime} \mathrm{px}^{-1}$. We use the continuum-subtracted $\mathrm{H} \alpha$ line image as a reference. From this image we create contours that highlight the most prominent morphological features in $\mathrm{H} \alpha$. We then produce a continuum-subtracted $\mathrm{H} \alpha$ map from the PMAS data cubes by subtracting a version of the data cube that is medianfiltered in spectral direction. Finally, we visually match the contours from the reference image with the PMAS H $\alpha$ map. This constrains the position of the PMAS FoV relative to the HST imaging. We emphasise that we make full use of the FITS header world-coordinate representation in our method, i.e. the headers of our final data cubes are equipped with keyword value pairs to determine the position of each spaxel on the sky (Greisen \& Calabretta 2002; Calabretta \& Greisen 2002).

For galaxies with a single PMAS pointing, we present the final result of our registration procedure in Fig. 5 (or Figs. 6 and 7, for the two galaxies with two PMAS pointings). For each LARS galaxy, the $\mathrm{H} \alpha$ line intensity map extracted from our HST observations is shown in the top panel and can be compared to the PMAS H $\alpha$ signal-to-noise ratio (S/N) map in the panel below. The $\mathrm{S} / \mathrm{N}$ values for each spaxel are calculated by summation of all flux values within a narrow spectral window centred on $\mathrm{H} \alpha$ and subsequent division by the square root of the sum of the variance values in that window. The width of the summation window is taken as twice the $\mathrm{H} \alpha$ lines $F W H M$. Our display of the HST $\mathrm{H} \alpha$ images uses an asinh-scaling (Lupton et al. 2004) cut at $95 \%$ of the maximum value (see Sect. 4 and Fig. 3 of Hayes et al. 2014, for absolute $\mathrm{H} \alpha$ intensities) and we scaled our PMAS $\mathrm{H} \alpha \mathrm{S} / \mathrm{N}$ maps logarithmically. The inferred final position of the PMAS FoV is indicated with a white square (or two squares for the galaxies with two pointings) within the $\mathrm{H} \alpha$ panel. Also shown are the $\mathrm{H} \alpha$ contours used for visual matching. As can be seen, most of the prominent morphological characteristics present in the HST H $\alpha$ maps are unambiguously identifiable in the lower resolution PMAS maps, exemplifying the robustness of our registration method.

\section{Ancillary LARS data products}

We compare our PMAS data to the HST imaging results of the LARS project presented in Hayes et al. (2013) and Paper II. Specifically, we use the continuum-subtracted $\mathrm{H} \alpha$ and $\operatorname{Ly} \alpha$ images that were presented in Paper II. These images were produced from our HST observation using the LARS extraction software LaXs (Hayes et al. 2009). Details on the observational strategy, reduction steps, and analysis performed to obtain these HST data products used in the present study are given in Paper II and Paper I.

We also compare with the published results of the LARS $\mathrm{HI}$ imaging and spectroscopy observations that were obtained with the $100 \mathrm{~m}$ Robert C. Byrd Green Bank Telescope (GBT) and the Karl G. Jansky Very Large Array (VLA). The GBT singledish spectra are present for all systems, except that the H I signal could not be detected in the three LARS galaxies with the largest distances (LARS 12, LARS 13, and LARS 14). The VLA interferometric imaging results are only available for LARS 2, LARS 3, LARS 4, LARS 8, and LARS 9. We emphasise that with beam sizes from $59^{\prime \prime}$ to $72^{\prime \prime}$ (VLA D-configuration) the
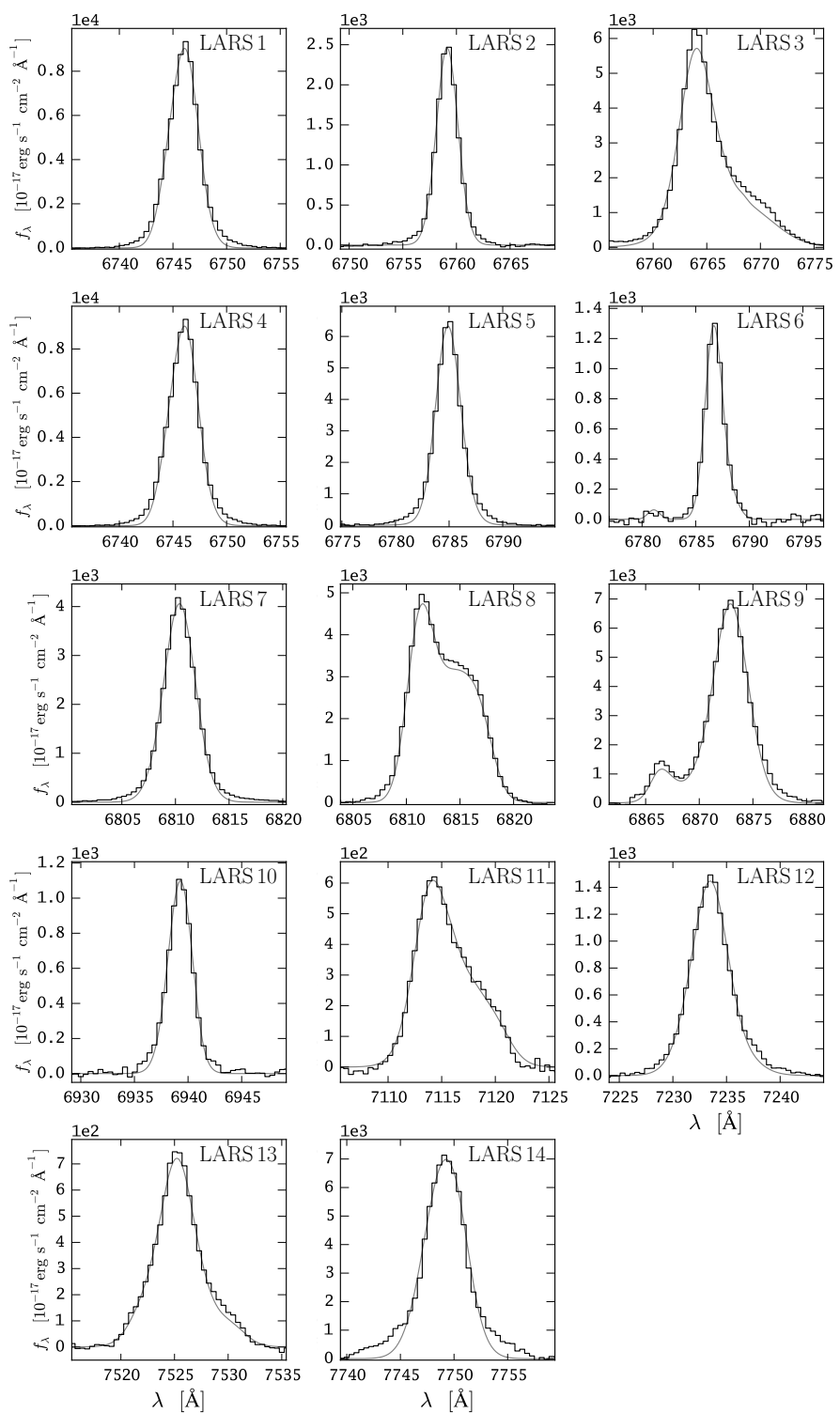

$\lambda[\AA]$

Fig. 3. Integrated continuum-subtracted $\mathrm{H} \alpha$ profiles of the LARS galaxies (black) compared to summed 1D Gaussian model profiles (grey) from which radial-velocity and velocity-dispersion maps were generated.

spatial scale that is resolved within the VLA H I images is much larger than our PMAS observations. Moreover, the single-dish GBT spectra are sensitive to $\mathrm{H}$ I at the observed frequency range within $8^{\prime}$ of the beam. Full details on data acquisition, reduction, and analysis are presented in Paper III.

\section{Analysis and results}

\subsection{Ho velocity fields}

We condense the kinematical information traced by $\mathrm{H} \alpha$ in our data cubes into two-dimensional maps depicting velocity dispersion and line-of-sight radial velocity at each spaxel. Both quantities are derived from 1D Gaussian fits to the observed line shapes from the continuum-subtracted cube (see Sect. 3.4). We calculate the radial velocity offset $v_{\mathrm{LOS}}$ to the central wavelength value given by the mean of all fits and the FWHM of the fitted 

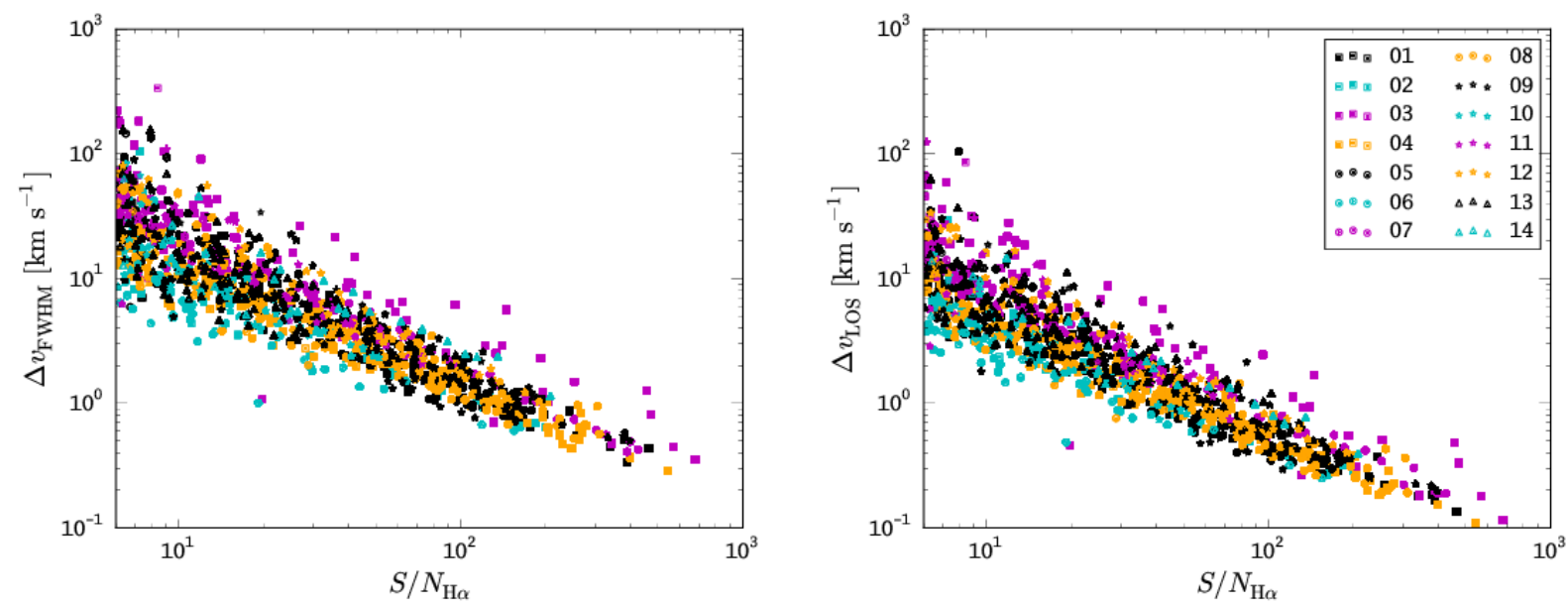

Fig. 4. Uncertainties on derived velocity dispersions (left panel) and radial velocities (right panel) for Gaussian profile fits to H $\alpha$ for all galaxies. All uncertainties for a specific galaxy have the same symbol according to the legend in the right panel.

line in velocity space $v_{\text {FWHM }}$. To obtain higher $\mathrm{S} / \mathrm{N}$ ratios in the galaxies' outskirts we use the weighted Voronoi tessellation binning algorithm by Diehl \& Statler (2006), which is a generalisation of the Voronoi binning algorithm of Cappellari \& Copin (2003).

We visually scrutinised the fits to the spectra and decided that lines observed at a $\mathrm{S} / \mathrm{N}$ of six were still reliably fit. Hence, six defines the minimum $\mathrm{S} / \mathrm{N}$ in the Voronoi binning algorithm. Furthermore, we adopted a maximum bin size of $3 \times 3$ pixels. In those calculations signal and noise are defined as in Sect. 3.4. In what follows, only results from the fits to bins that meet the minimum $\mathrm{S} / \mathrm{N}$ requirement are considered. Most of the bins used are actually only a single spaxel, and very few bins in the outskirts of the galaxies meeting the minimum $\mathrm{S} / \mathrm{N}$ criterion do consist of 2 or 3 spaxels. In practice this means that we are typically sensitive to regions at $\mathrm{H} \alpha$ surface brightness higher than $5 \times$ $10^{-16} \mathrm{erg} \mathrm{s}^{-1} \mathrm{~cm}^{-2} \operatorname{arcsec}^{-2}$. Using the Kennicutt (1998) conversion and neglecting extinction, this translates to a detection limit of star-formation rate surface densities $\sim 3 \times 10^{-2} M_{\odot} \mathrm{yr}^{-1} \mathrm{kpc}^{-2}$, more than an order of magnitude deeper than probed by high redshift integral field spectroscopy studies $\left(\sim 1 M_{\odot} \mathrm{yr}^{-1} \mathrm{kpc}^{-2}\right.$, see e.g. Law et al. 2009).

By visual inspection we also ensured that 1D Gaussian profiles are a sufficient model of the observed $\mathrm{H} \alpha$ profiles seen in the PMAS LARS data cubes. We only have some spaxels where a 1D Gaussian certainly fails to reproduce the complexity of the observed spectral shape in two galaxies (LARS 9 and LARS 13). Moreover, in LARS 14 weak broad wings are visible in the individual $\mathrm{H} \alpha$ profiles. We have not attempted to model these special cases, but we describe their qualitative appearance in detail in the Appendices A.9, A.13, and A.14, and acknowledge their existence in our interpretation in Sect. 6. To demonstrate the overall quality of our $\mathrm{H} \alpha$ velocity fields, we compare in Fig. 3 the integrated $\mathrm{H} \alpha$ profiles of the LARS galaxies to the sum of the fitted $1 \mathrm{D}$ Gaussians used to generate the $v_{\mathrm{FWHM}}$ and $v_{\mathrm{LOS}}$ maps. As can be seen, the observed profile and that derived from the models are largely in agreement. The strongest deviation between data and models is apparent in LARS 14, where the broad wings of the observed $\mathrm{H} \alpha$ profile are not reproduced at all by 1D Gaussian models (cf. Sect. 6.1 and Appendix A.14).

To estimate formal uncertainties on $v_{\text {FWHM }}$ and $v_{\text {LOS }}$, we use a Monte Carlo technique: we perturb our spectra 100 times with the noise from our noise cubes and then fit the 1D Gaussian profile in exactly the same way to each of these 100 realisations. The width of the distribution of all fit results characterised by their standard deviation gives a robust measure for the uncertainty on the derived quantities (cf. Appendix B.4. in Davies et al. 2011). The central moment of the distribution is the final value that is shown in our maps. In Fig. 4 we indicate, for all performed fits, the error on the velocity dispersion $\Delta v_{\mathrm{FWHM}}$ and on radial velocity $\Delta v_{\mathrm{LOS}}$ as a function of the lines $\mathrm{S} / \mathrm{N}$. On average our uncertainties on $v_{\mathrm{FWHM}}$ and $v_{\mathrm{LOS}}$ follow the scaling laws expected for fitting Gaussian profiles to noisy emission-line spectra $\Delta v_{\mathrm{LOS}} \propto \Delta v_{\mathrm{FWHM}} \propto v_{\mathrm{FWHM}}^{1 / 2}(S / N)^{-1}$ (Landman et al. 1982; Lenz $\&$ Ayres 1992). For reference, at our minimum $S / N=6$ we have typical errors of $\Delta v_{\mathrm{FWHM}} \approx 30 \mathrm{~km} \mathrm{~s}^{-1}$ and $\Delta v_{\mathrm{LOS}} \approx 10 \mathrm{~km} \mathrm{~s}^{-1}$, and at the median (mean) $\mathrm{S} / \mathrm{N}$ of our data $-S / N_{\text {median }}=22$ $\left(S / N_{\text {mean }}=50\right)$, we obtain $\Delta v_{\mathrm{FWHM}} \approx 7 \mathrm{~km} \mathrm{~s}^{-1}\left(\approx 3 \mathrm{~km} \mathrm{~s}^{-1}\right)$ and $\Delta v_{\text {LOS }} \approx 3 \mathrm{~km} \mathrm{~s}^{-1}\left(\approx 1 \mathrm{~km} \mathrm{~s}^{-1}\right)$.

In Fig. 5 we show the resulting $v_{\text {FWHM }}$ and $v_{\text {LOS }}$ maps together with the LaXs Ly $\alpha$ images (lower three panels). Maps and images of the galaxies observed with two pointings are shown in Figs. 6 and 7 for LARS 9 and LARS 13, respectively. All $v_{\text {FWHM }}$ maps have been corrected for instrumental broadening using the spectral resolving power maps derived in Sect. 3.3. In all $v_{\mathrm{LOS}}$ and $v_{\text {FWHM }}$ panels, we overlay contours derived from the Ly $\alpha$ image to emphasise distinctive morphological Ly $\alpha$ properties.

Individual maps are discussed in Appendix A. Using the classification scheme introduced by Flores et al. (2006; see also Sect. 3.3 in Glazebrook 2013) the LARS galaxies can be characterised by the qualitative appearance of their velocity fields:

- Rotating disks: LARS 8 and LARS 11. Both galaxies show a regular symmetric dipolar velocity field with a steep gradient in the central regions. This steep gradient is also the reason for artificially broadened emission near the kinematical centre. Morphologically both galaxies also bear resemblance to a disk and the kinematical axis is aligned with the morphological axis. However, we point out in Appendix A.8 that LARS 8 can also be classified as a shell galaxy, hinting at a recent merger event.

- Perturbed rotators: LARS 1, LARS 3, LARS 5, LARS 7, and LARS 10. In those galaxies traces of orbital motion are still noticeable, but the maps look either significantly perturbed compared to a classical disk case (LARS 3, LARS 5, 

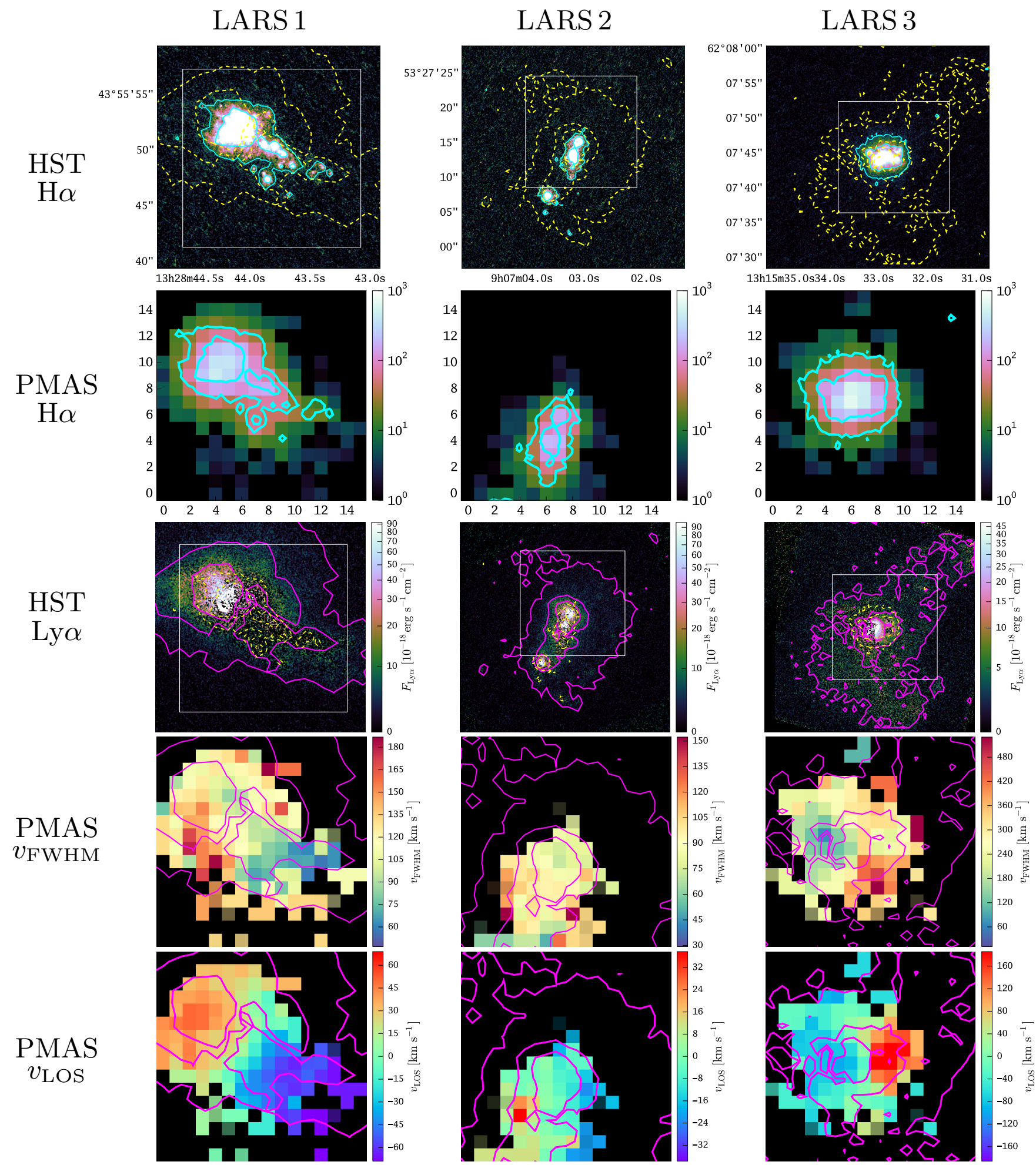

Fig. 5. Comparison of LARS HST imaging results of the LARS sample to spatially resolved PMAS H $\alpha$ spectroscopy. North is always up and east is always to the left. For each galaxy from top to bottom: the first panel shows the LaXs $\mathrm{H} \alpha$ line intensity map; tick labels indicate right ascension and declination and an asinh-scaling is used cut at $95 \%$ of the maximum value. The second panel shows a S/N map of the continuum-subtracted $\mathrm{H} \alpha$ signal observed with PMAS. Tick labels in the PMAS S/N map are in arc-seconds; the scaling is logarithmic from 1 to $10^{3}$ and only spaxels with $S / N>1$ are shown. The third panel shows the LARS Ly $\alpha$ images with a colour bar indicating the flux scale in cgs-units; scaling is the same as in the $\mathrm{H} \alpha$ map. The fourth panel shows resolution-corrected $\mathrm{H} \alpha v_{\text {FWHM }}$ maps from our PMAS observations and the corresponding $\mathrm{H} \alpha v_{\mathrm{LOS}}$ maps are shown in the fifth panel. In the first and third panel, we indicate the position and extent of the PMAS field of view with a white box. Cyan contours in the HST H $\alpha$ image are contours of constant surface brightness, adjusted to highlight the most prominent morphological features. Similarly, magenta contours in the HST Ly $\alpha$ images indicate the Ly $\alpha$ morphology; these contours are also shown in the fourth and fifth panel. To highlight the difference between $\operatorname{Ly} \alpha$ and $\mathrm{H} \alpha$, the $\operatorname{Ly} \alpha$ and $\mathrm{H} \alpha$ panels contain as dashed yellow lines the contours from the $\mathrm{H} \alpha$ and $\mathrm{Ly} \alpha$ panels, respectively. 


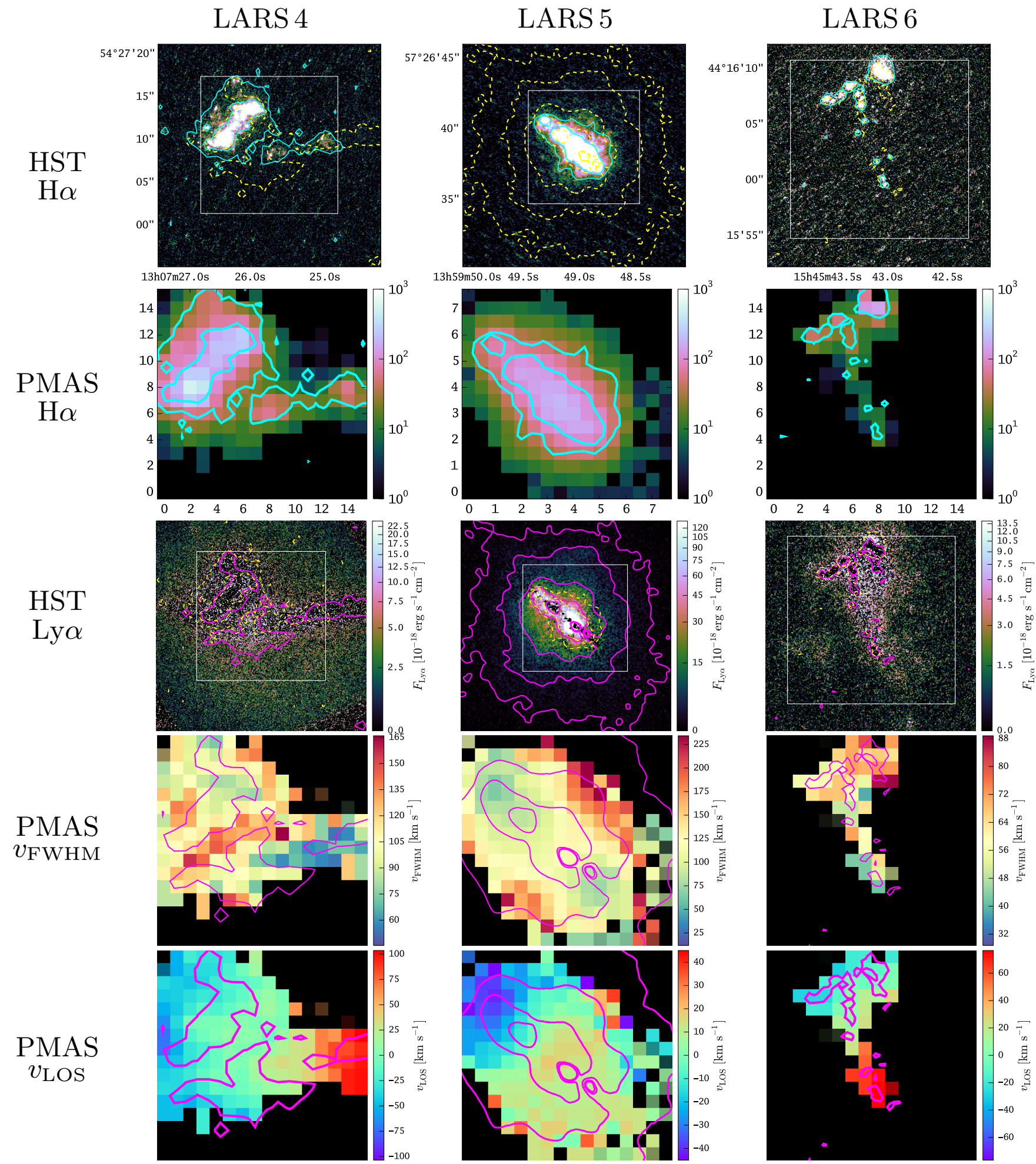

Fig. 5. continued.

LARS 7, and LARS 10) and/or the observed velocity gradient is very weak (LARS 1, LARS 5 and LARS 10). Based solely on morphology, we previously classified two of those galaxies as dwarf edge-on disks (LARS 5 and LARS 7; Paper II; Paper III). Moreover, LARS 3 is a member of a well known merger of two similar massive disks (see also Appendix A.3). From our imaging data alone LARS 7 and LARS 10 bear resemblance to shell galaxies (see also Appendices A.7 and A.10).

- Complex kinematics: LARS 2, LARS 4, LARS 6, LARS 9, LARS 12, LARS 13, and LARS 14. These seven galaxies are characterised by more chaotic $v_{\mathrm{LOS}}$ maps and they all 

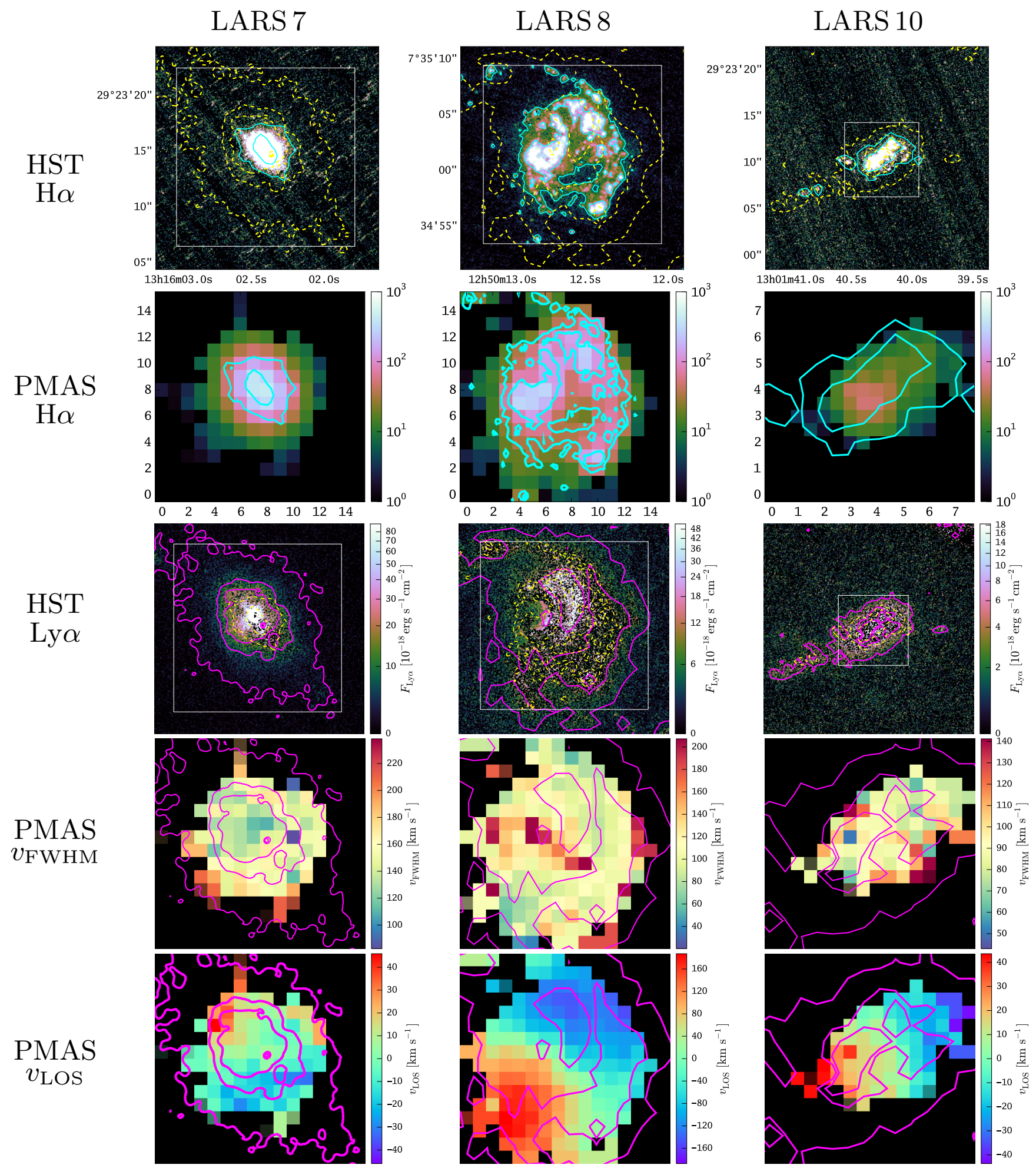

Fig. 5. continued.

have also irregular morphologies. Four of the irregulars consist of photometrically well-separated components that are also kinematically distinct (LARS 4, LARS 6, LARS13 and LARS 14), while the other three each have their own individual peculiarities (LARS 2, LARS 9 and most spectacularly LARS 12 - see also Appendices A.2, A.9, and A.12).

These kinematic classes are also listed in Table 2.
Notably, in all LARS galaxies, except for LARS 6, we observe high-velocity dispersions $\left(v_{\mathrm{FWHM}} \gtrsim 100 \mathrm{~km} \mathrm{~s}^{-1}\right)$; the most extreme case is LARS 3 where locally lines as broad as $v_{\text {FWHM }} \sim 400 \mathrm{~km} \mathrm{~s}^{-1}$ are found. The observed width of the $\mathrm{H} \alpha$ line seen in our $v_{\mathrm{FWHM}}$ maps can be described as a successive convolution of the natural $\mathrm{H} \alpha$ line $\left(7 \mathrm{~km} \mathrm{~s}^{-1}\right.$ FHWM) with the thermal velocity distribution of the ionised gas $\left(21.4 \mathrm{~km} \mathrm{~s}^{-1}\right.$ FWHM at $10^{4} \mathrm{~K}$ ) and with non-thermal motions in the gas 


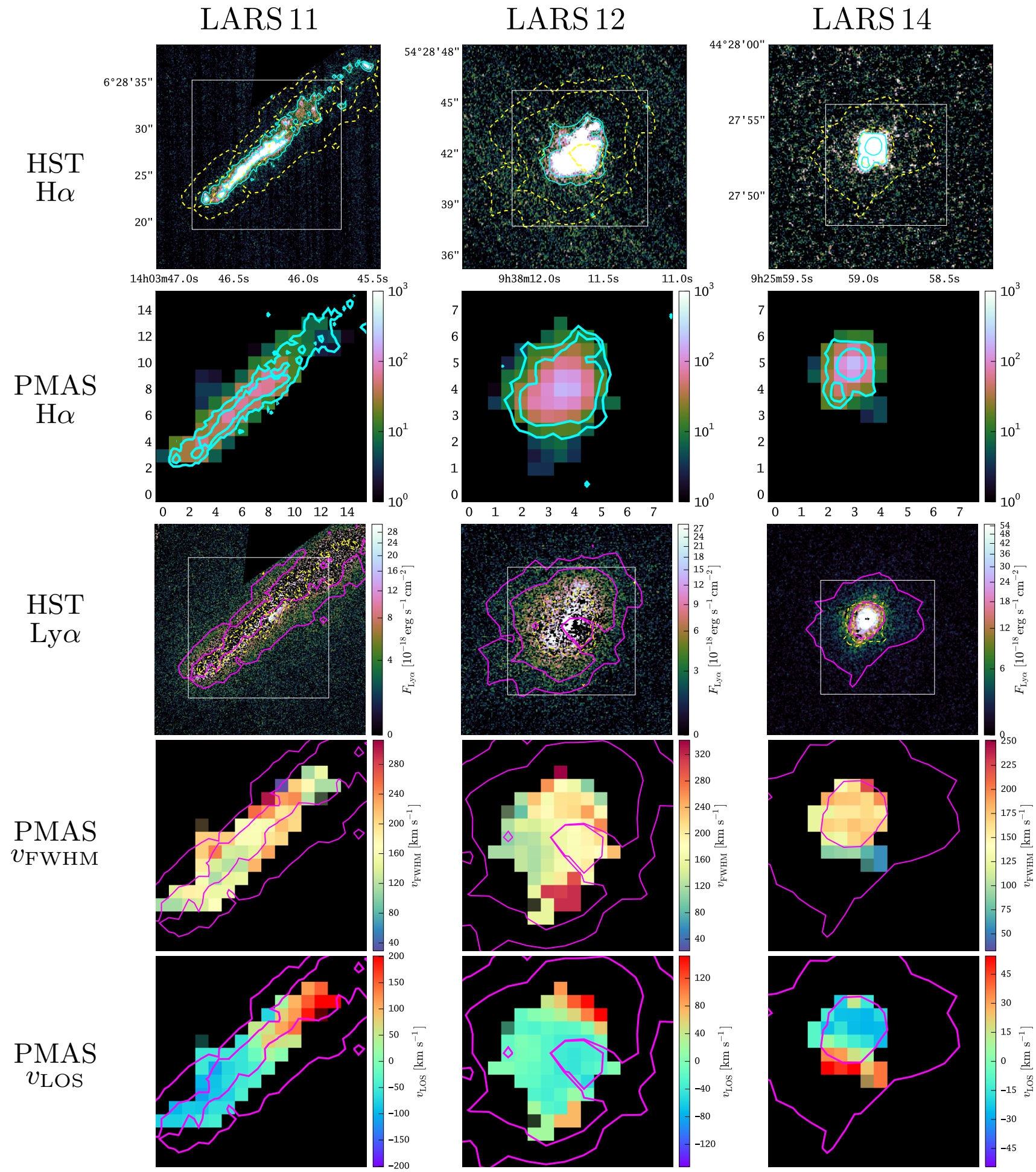

Fig. 5. continued.

(e.g. Jiménez-Vicente et al. 1999). Since all our dispersion measurements are significantly higher than the thermally broadened profile non-thermal motions dominate the observed linewidths. Non-thermal motions could be centre-to-centre dispersions of the individual $\mathrm{H}$ II regions and turbulent motions of the ionised gas. The latter appears to be the main driver for the observed line widths, since the observed velocity dispersions are highly supersonic (i.e. $\gtrsim 10 \mathrm{~km} \mathrm{~s}^{-1}$ for $\mathrm{H} \mathrm{II}$ at $10^{4} \mathrm{~K}$; see also Sect. 5.1 in Glazebrook 2013).

\subsection{Global kinematical properties: $v_{\text {shear }}, \sigma_{0}, \sigma_{\text {tot }}$ and $v_{\text {shear }} / \sigma_{0}$}

We now quantify the global kinematical properties of our $\mathrm{H} \alpha$ velocity fields that were qualitatively discussed in the previous section. Therefore, we compute three non-parametric estimators that are commonly adopted in the literature for this purpose (e.g. Law et al. 2009; Basu-Zych et al. 2009; Green et al. 2010; Gonçalves et al. 2010; Glazebrook 2013; Green et al. 2014; Wisnioski et al. 2015): shearing velocity $v_{\text {shear }}$, intrinsic velocity 


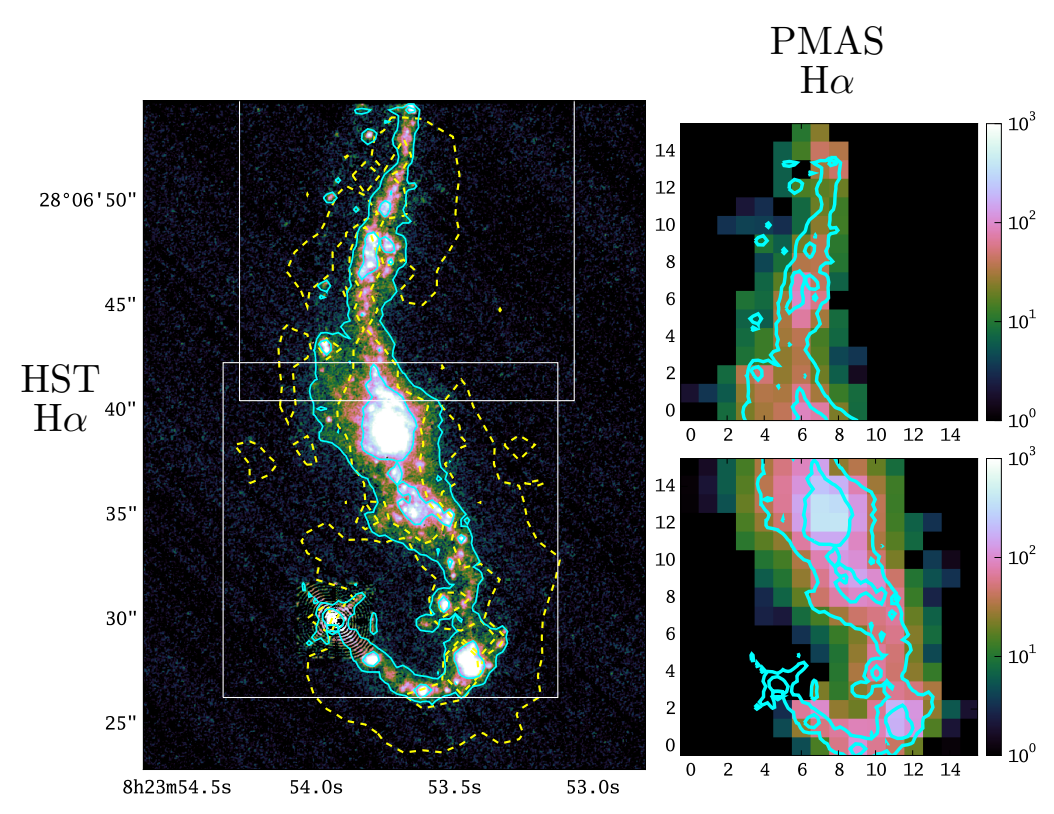

\section{LARS 9}
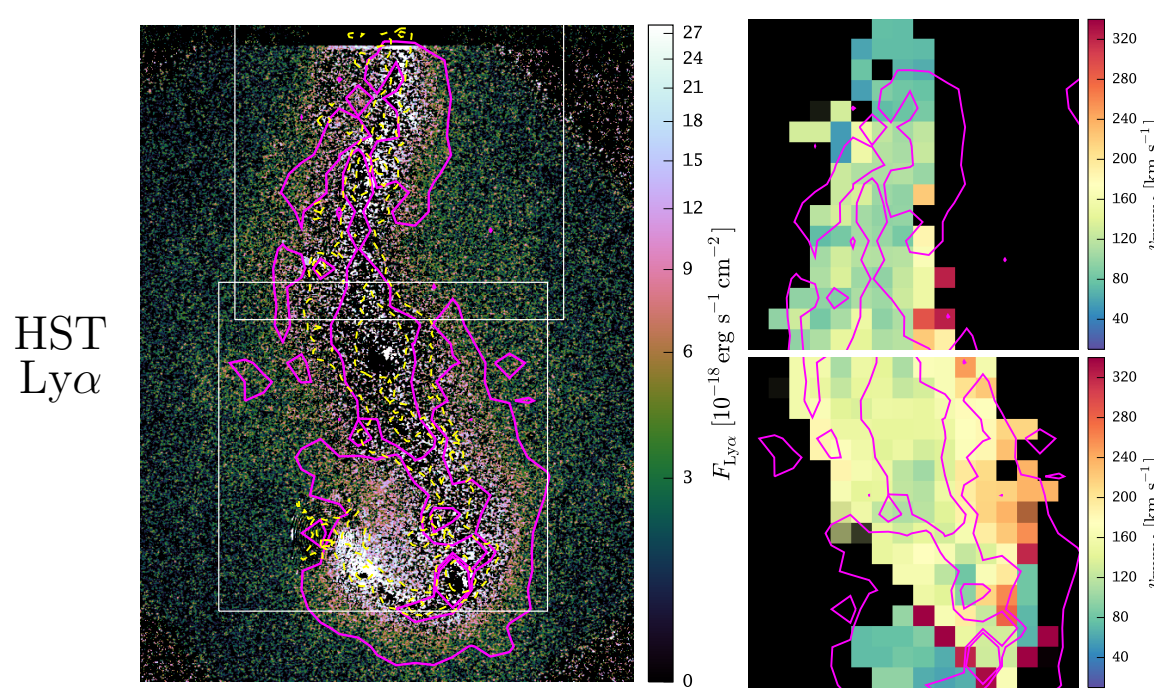

PMAS

$v_{\text {FWHM }}$

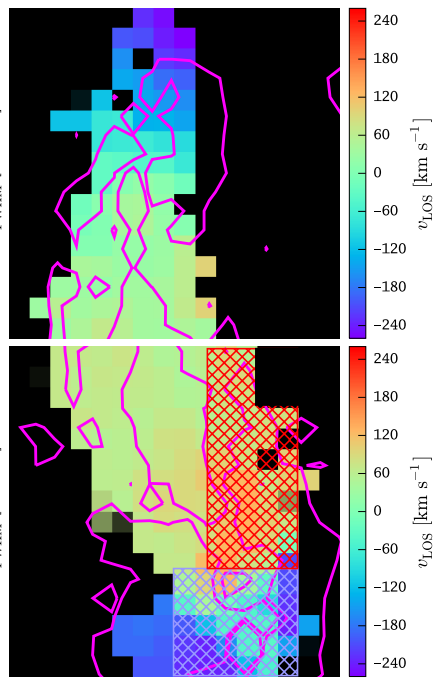

PMAS

$v_{\mathrm{LOS}}$

Fig. 6. Comparison of LARS HST imaging results to spatially resolved PMAS H $\alpha$ spectroscopy for LARS 9. For detailed description of individual panels see caption of Fig. 5. This galaxy was covered with 2 PMAS pointings. Hatched regions in the $v_{\text {LOS }}$ map indicate regions, where the H $\alpha$ emission shows a more complex profile that could not be described by a simple Gaussian (see also Appendix A.9).

dispersion $\sigma_{0}$, and their ratio $v_{\text {shear }} / \sigma_{0}$. We describe the physical meaning of those parameters and our method to determine them in the following three subsections. Furthermore, we also calculate the integrated velocity dispersion $\sigma_{\text {tot }}$. This measure provides a useful comparison for unresolved distant galaxies. Our results on $v_{\text {shear }}, \sigma_{0}, \sigma_{\text {tot }}$ and $v_{\text {shear }} / \sigma_{0}$ are tabulated in Table 2 .

\subsubsection{Shearing velocity $v_{\text {shear }}$}

The shearing velocity $v_{\text {shear }}$ is a measure for the large-scale gas bulk motion along the line of sight. We calculate it via

$v_{\text {shear }}=\frac{1}{2}\left(v_{\max }-v_{\min }\right)$.
Here we take the median of the lower and upper fifth percentile of the distribution of values in the $v_{\mathrm{LOS}}$ maps for $v_{\min }$ and $v_{\max }$, respectively. This choice ensures that the calculation is robust against outliers while sampling the true extremes of the distribution. We conservatively estimate the uncertainty by propagating the full width of the upper and lower fifth percentile of the velocity map. We list our results for $v_{\text {shear }}$ in the second column of Table 2. Our derived $v_{\text {shear }}$ values for the LARS sample range from $30 \mathrm{~km} \mathrm{~s}^{-1}$ to $180 \mathrm{~km} \mathrm{~s}^{-1}$, with $65 \mathrm{~km} \mathrm{~s}^{-1}$ being the median. This is less than half the typical maximum velocity $v_{\max }$ of $\mathrm{H} \alpha$ rotation curves observed in spiral galaxies, e.g. $v_{\max }=145 \mathrm{~km} \mathrm{~s}^{-1}$ is the median of 153 local spirals (Epinat et al. 2010). In contrast to our $v_{\text {shear }}$ measurement, $v_{\max }$ values are inclination corrected. In a sample with random inclinations on average the correction $v_{\text {shear }}=\frac{\pi}{4} v_{\max } \approx 0.79 v_{\max }$ is expected 

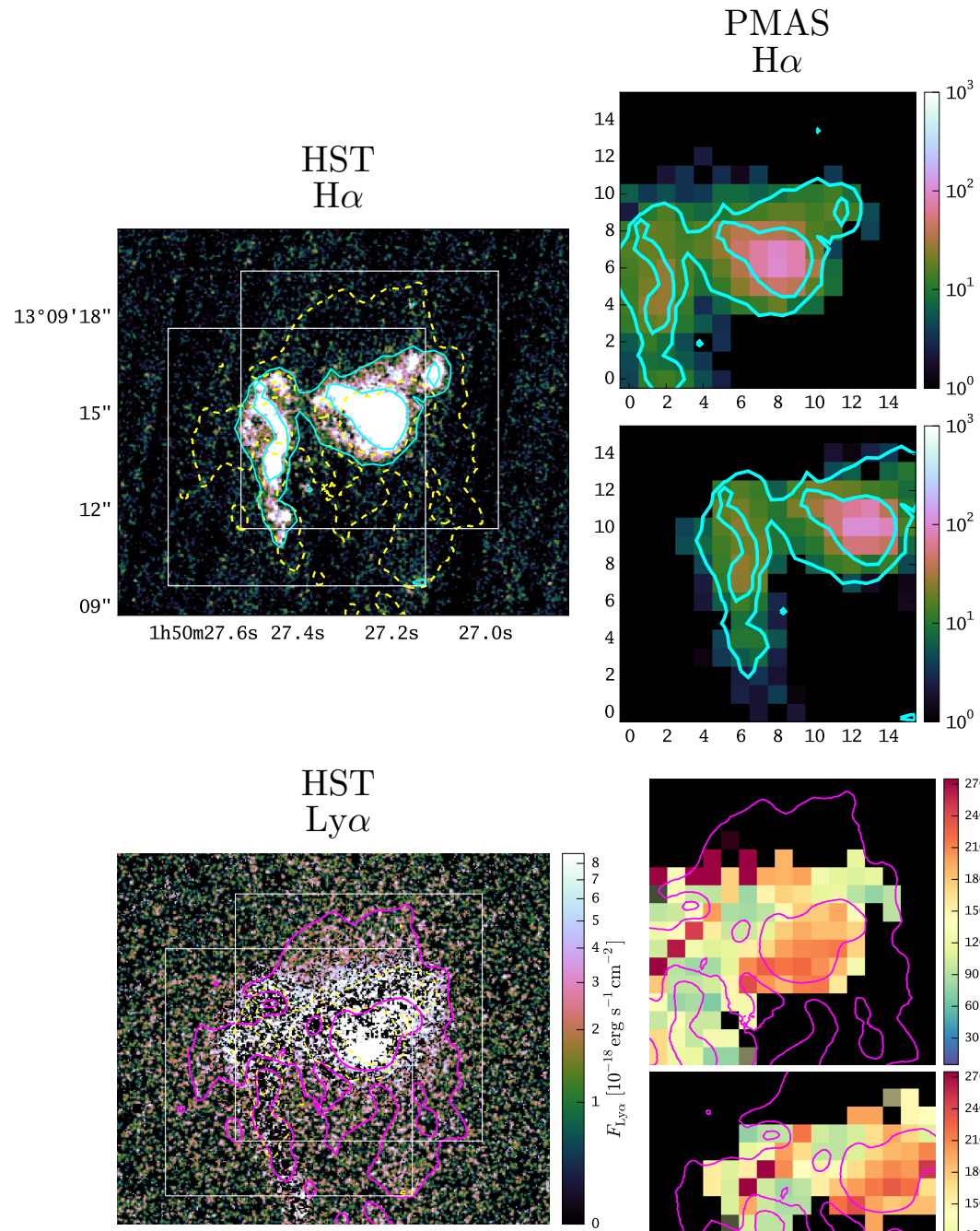

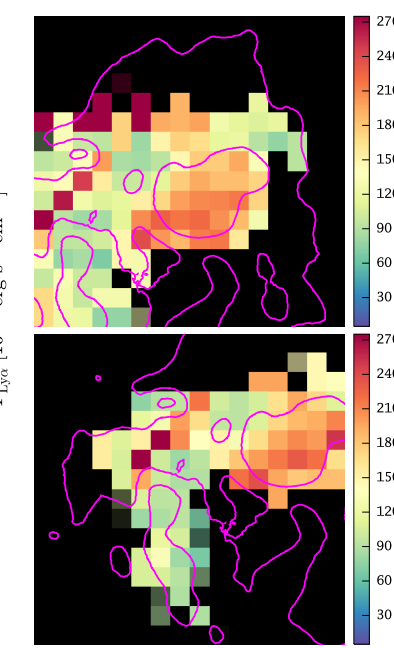

PMAS

$v_{\text {FWHM }}$

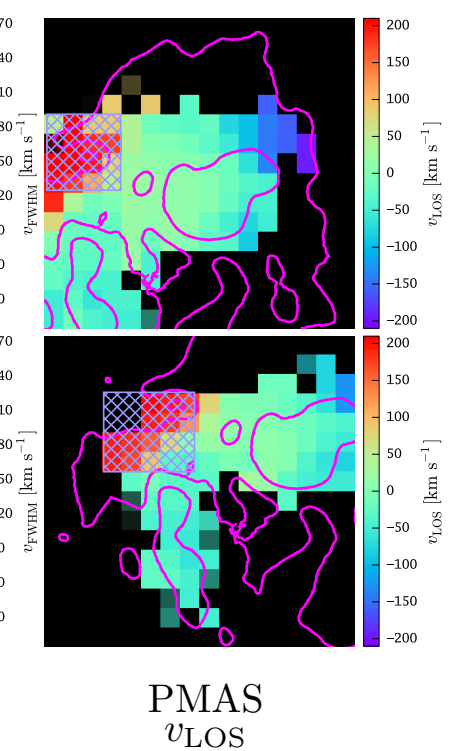

Fig. 7. Same as Fig. 6, but for LARS 13. Hatched region in the $v_{\text {LOS }}$ map indicates the region, where the H $\alpha$ emission shows a more complex profile that could not be described by a simple Gaussian (see also Appendix A.13).

(e.g. Law et al. 2009), but even with this correction classical disks appear still incompatible with most of our measurements by a factor larger than two. We only find $v_{\text {shear }}$ values compatible with local rotators for the two galaxies that we classified as rotating disks in Sect. 5.1. Nevertheless, high $v_{\text {shear }}$ values do not necessarily imply the presence of a disk. Large-scale bulk motions at high amplitude also occur in close encounters of spatially and kinematically distinct companions (e.g. LARS 13).

Sensitivity is an important factor in the determination of the observed $v_{\text {shear }}$. An observation of less depth does not detect fainter regions at larger radii, which may have the highest velocities. Indeed, for most of our galaxies the spatial positions of $v_{\min }$ and $v_{\max }$ values are in those outer lower surface-brightness regions. Hence observations of lower sensitivity are biased to lower $v_{\text {shear }}$ values. This sensitivity bias is strongly affecting high $-z$ studies because these kinds of observations are often only limited to the brightest regions (Law et al. 2009; Gonçalves et al. 2010).

\subsubsection{Intrinsic velocity dispersion $\sigma_{0}$}

The intrinsic velocity dispersion $\sigma_{0}$ (sometimes also called resolved velocity dispersion or local velocity dispersion, cf. Glazebrook 2013, Sect. 1.2.1) is in our case a measure for the typical random motions of the ionised gas within a galaxy. We calculate it by taking the flux weighted average of the observed velocity dispersions in all spaxels, i.e.

$$
\sigma_{0}=\frac{\sum F_{\text {spaxel }}^{\mathrm{H} \alpha} \sigma_{\text {spaxel }}}{\sum F_{\text {spaxel }}^{\mathrm{H} \alpha}}
$$

Here $F_{\text {spaxel }}^{\mathrm{H} \alpha}$ is the $\mathrm{H} \alpha$ flux in each spaxel and $\sigma_{\text {spaxel }}$ is the velocity dispersion measured in that spaxel; the sum runs over all spaxels with $S / N \geq 6$ (Sect. 5.1). This kind of flux-weighted summation $\sigma_{0}$ has been widely adopted in the literature to quantify the typical intrinsic velocity dispersion component 
A\&A 587, A78 (2016)

Table 2. Global kinematic parameters from $\mathrm{H} \alpha$ for the LARS galaxies, calculated as described in Sect. 5.2.

\begin{tabular}{cccrrrrrrc}
\hline \hline ID & Class & $\begin{array}{c}\sigma_{0} \\
{\left[\mathrm{~km} \mathrm{~s}^{-1}\right]}\end{array}$ & $\begin{array}{c}\sigma_{\text {tot }}\left[\mathrm{km} \mathrm{s}^{-1}\right] \\
{\left[\mathrm{km} \mathrm{s}^{-1}\right]}\end{array}$ & $\begin{array}{c}v_{\text {shear }} / \sigma_{0} \\
E W_{\text {Ly } \alpha} \\
{[\AA]}\end{array}$ & $\begin{array}{c}\text { Ly } \alpha / \mathrm{H} \alpha \\
f_{\text {esc. }}^{\text {Ly }}\end{array}$ & $\xi_{\text {Ly } \alpha}$ \\
\hline 1 & $\mathrm{P}$ & $47.5 \pm 0.1$ & 60.5 & $56 \pm 2$ & $1.2 \pm 0.1$ & 33.0 & 1.36 & 0.119 & 3.37 \\
2 & $\mathrm{C}$ & $38.6 \pm 0.9$ & 46.0 & $23 \pm 3$ & $0.6 \pm 0.1$ & 81.7 & 4.53 & 0.521 & 2.27 \\
3 & $\mathrm{P}$ & $99.5 \pm 3.7$ & 130.9 & $138 \pm 3$ & $1.4 \pm 0.2$ & 16.3 & 0.16 & 0.003 & 0.77 \\
4 & $\mathrm{C}$ & $44.1 \pm 0.1$ & 55.1 & $74 \pm 9$ & $1.7 \pm 0.1$ & 0.00 & 0.00 & 0.000 & $\ldots$ \\
5 & $\mathrm{P}$ & $46.8 \pm 0.3$ & 54.7 & $37 \pm 4$ & $0.8 \pm 0.1$ & 35.9 & 2.16 & 0.174 & 2.61 \\
6 & $\mathrm{C}$ & $27.2 \pm 0.3$ & 58.9 & $52 \pm 7$ & $1.9 \pm 0.2$ & 0.00 & 0.00 & 0.000 & $\ldots$ \\
7 & $\mathrm{P}$ & $58.7 \pm 0.3$ & 67.4 & $31 \pm 3$ & $0.5 \pm 0.1$ & 40.9 & 1.94 & 0.100 & 3.37 \\
8 & $\mathrm{R}$ & $49.0 \pm 0.1$ & 111.8 & $155 \pm 3$ & $3.2 \pm 0.1$ & 22.3 & 0.67 & 0.025 & 1.12 \\
9 & $\mathrm{C}$ & $58.6 \pm 0.1$ & 112.7 & $182 \pm 3$ & $3.1 \pm 0.1$ & 3.31 & 0.13 & 0.007 & $>2.85$ \\
10 & $\mathrm{P}$ & $38.2 \pm 1.0$ & 49.2 & $36 \pm 3$ & $0.9 \pm 0.2$ & 8.90 & 0.47 & 0.026 & 2.08 \\
11 & $\mathrm{R}$ & $69.3 \pm 3.8$ & 114.8 & $149 \pm 4$ & $2.1 \pm 0.3$ & 7.38 & 0.72 & 0.036 & 2.27 \\
12 & $\mathrm{C}$ & $72.7 \pm 1.0$ & 81.3 & $96 \pm 3$ & $1.3 \pm 0.1$ & 8.49 & 0.48 & 0.009 & 3.48 \\
13 & $\mathrm{C}$ & $69.2 \pm 0.7$ & 97.3 & $183 \pm 4$ & $2.5 \pm 0.2$ & 6.06 & 0.29 & 0.010 & 1.74 \\
14 & $\mathrm{C}$ & $67.3 \pm 1.3$ & 72.9 & $40 \pm 1$ & $0.6 \pm 0.1$ & 39.4 & 2.24 & 0.163 & 3.62 \\
\hline
\end{tabular}

Notes. For reference we also tabulate $E W_{\mathrm{Ly} \alpha}, \mathrm{Ly} \alpha / \mathrm{H} \alpha$ and $f_{\mathrm{esc}}^{\mathrm{Ly} \alpha}$ from Paper II, as well as $\xi_{\mathrm{Ly} \alpha}$ from Hayes et al. (2013). Class refers to the three kinematic classes proposed by Flores et al. (2006): $\mathrm{C}=$ complex kinematics, $\mathrm{P}=$ perturbed rotators, and $\mathrm{R}=$ rotating disks.

(e.g. Östlin et al. 2001; Law et al. 2009; Gonçalves et al. 2010; Green et al. 2010, 2014; Glazebrook 2013).

The PSF smearing in the presence of strong velocity gradients broadens the $\mathrm{H} \alpha$ line at the position of the gradients ${ }^{9}$. This kind of broadening could bias our calculation of the intrinsic velocity dispersion. At the distances of the LARS galaxies, our typical seeing-disk PSF FWHM of 1" corresponds to scales of $0.6 \mathrm{kpc}$ to $3 \mathrm{kpc}$. In most of our observations, this average PSF FWHM is comparable to the size of one PMAS spaxel $\left(1^{\prime \prime} \times 1^{\prime \prime}\right)$ and most of the LARS galaxies exhibit rather weak velocity gradients. The PSF smearing effect is only seen in galaxies with strong velocity gradients (e.g. LARS 8; see also Appendix A.8), especially when observed with PMAS' $0.5^{\prime \prime} \times 0.5^{\prime \prime}$ magnification mode (e.g. LARS 12; see also Appendix A.12). In principle, the effect could be corrected by subtracting a PSF-smeared kinematical model of the $v_{\text {LOS }}$ velocity field. Given the complexity of our observed velocity fields, however, this is not practicable. And, moreover, at $z \sim 0.1$ Green et al. (2014) find that for classical rotating disk kinematics the velocity dispersions corrected by a kinematical model are, on average, negligible. Comparisons between adaptive-optics derived PSF-smearing unaffected $\sigma_{0}$ values to those derived from seeing-limited observations were presented by Bassett et al. (2014). They find that while for one galaxy $\sigma_{0}$ remains unaffected by an increase in spatial resolution, for the other galaxy $\sigma_{0}$ decreases by $\sim 10 \mathrm{~km} \mathrm{~s}^{-1}$. Bassett et al. (2014) attribute the $\sim 10 \mathrm{~km} \mathrm{~s}^{-1}$ discrepancy in one of their galaxies to a strong velocity gradient that is co-spatial with the strongest emission. A similar comparison is possible for three of our LARS galaxies - LARS 12, LARS 13 and LARS 14. For these galaxies adaptive optics IFS observations were presented by Gonçalves et al. (2010) (see Appendices A.12-A.14). Gonçalves et al. (2010) derive $\sigma_{0}=67 \mathrm{~km} \mathrm{~s}^{-1}, \sigma_{0}=74 \mathrm{~km} \mathrm{~s}^{-1}$ and $\sigma_{0}=71 \mathrm{~km} \mathrm{~s}^{-1}$ for LARS 12, LARS 13, and LARS 14 , respectively. These values are in agreement with our measurements of $\sigma_{0}=73 \mathrm{~km} \mathrm{~s}^{-1}, \sigma_{0}=69 \mathrm{~km} \mathrm{~s}^{-1}$, and $\sigma_{0}=$ $67 \mathrm{~km} \mathrm{~s}^{-1}$ for those galaxies ${ }^{10}$. From all these considerations we

\footnotetext{
9 This effect is similar to beam smearing in radio interferometric imaging observations.

${ }^{10}$ For LARS 13, Gonçalves et al. (2010) do not sample the entire galaxy in their observations; see Sect. A.13.
}

are certain, that the adopted formalism in Eq. (2) gives a robust estimate of the intrinsic velocity dispersion.

We list our derived values for $\sigma_{0}$ in the third column of Table 2. In general, the ionised gas kinematics of the LARS galaxies are characterised by high intrinsic velocity dispersions ranging from $40 \mathrm{~km} \mathrm{~s}^{-1}$ to $100 \mathrm{~km} \mathrm{~s}^{-1}$ with $54 \mathrm{~km} \mathrm{~s}^{-1}$ being the median of the sample. Such high intrinsic dispersions are in contrast to H II velocity dispersions of $\sim 10-50 \mathrm{~km} \mathrm{~s}^{-1}$ typically found in local spirals (e.g. Epinat et al. 2008a,b, 2010; Erroz-Ferrer et al. 2015). In high- $z$, star-forming galaxies intrinsic dispersions $\gtrsim 50 \mathrm{~km} \mathrm{~s}^{-1}$ appear to be the norm (e.g. Puech et al. 2006; Genzel et al. 2006; Law et al. 2009; Förster Schreiber et al. 2009; Erb et al. 2014; Wisnioski et al. 2015). In the local $z \lesssim 0.1$ Universe, high intrinsic velocity dispersions are also found in the studies of blue compact galaxies (BCGs; Östlin et al. 1999, 2001), local Lyman break analogues (Basu-Zych et al. 2009; Gonçalves et al. 2010) and (ultra-)luminous infrared galaxies (e.g. Monreal-Ibero et al. 2010). Finally, $\sigma_{0}$ values of comparable amplitude are also common in a sample of 67 bright $\mathrm{H} \alpha$ emitters $\left(10^{40.6} \leq L_{\mathrm{H} \alpha} \leq 10^{42.6}\right)$ (Green et al. 2010, 2014). Indeed, Green et al. (2010) and Green et al. (2014) show that star formation rate (SFR) is positively correlated with intrinsic velocity dispersion. We confirm this trend among our LARS galaxies and discuss its implications on $\mathrm{Ly} \alpha$ observables in Sect. 6.3.

\subsection{3. $v_{\text {shear }} / \sigma_{0}$ ratio}

The ratio $v_{\text {shear }} / \sigma_{0}$ is a metric to quantify whether the gas kinematics are dominated by turbulent or ordered (in some cases orbital) motions. Our results for this quantity are listed in Table 2 . Given the above (Sects. 5.2.1 and 5.2.2) mentioned differences to disks, it appears cogent that our $v_{\text {shear }} / \sigma_{0}$ ratios (median 1.4, mean 1.6) are much smaller than typical disk $v_{\text {shear }} / \sigma_{0}$ ratios 4-8 (first to last quartile of the Epinat et al. sample).

Objects with $v_{\text {shear }} / \sigma_{0}<1$ are commonly labelled dispersion-dominated galaxies (Newman et al. 2013; Glazebrook 2013). According to this criterion, five galaxies of the 14 LARS sample are dispersion dominated: LARS 2, LARS 5, LARS 7, LARS 10, and LARS 14; three perturbed rotators and two with complex kinematics. Dispersion-dominated galaxies are frequently found in kinematic studies of high- $z$, 
star-forming galaxies. Local samples with a similar percentage $(\sim 35 \%)$ of dispersion-dominated galaxies are the blue compact galaxies studied by Östlin et al. (2001) or the Lyman break analogues studied by Gonçalves et al. (2010). In Sect. 6.3, we show that dispersion-dominated systems are more likely to have a significant fraction of Ly $\alpha$ photons escaping.

\subsubsection{Integrated velocity dispersion $\sigma_{\text {tot }}$}

For reference we also compute the integrated (spatially averaged) velocity dispersion $\sigma_{\text {tot }}$. This measure provides a useful comparison for studies of unresolved distant galaxies where no disentanglement between ordered and disordered, random motions are possible (e.g. McLinden et al. 2011; Guaita et al. 2013; McLinden et al. 2014; Rhoads et al. 2014; Erb et al. 2014).

We obtain $\sigma_{\text {tot }}$ by calculating the square root of the second central moment of the integrated $\mathrm{H} \alpha$ profiles shown in Fig. 3. The results are given in the fourth column of Table 2. Our integrated velocity dispersions for the LARS sample range from $46 \mathrm{~km} \mathrm{~s}^{-1}$ to $115 \mathrm{~km} \mathrm{~s}^{-1}$, with $70 \mathrm{~km} \mathrm{~s}^{-1}$ being the median of the sample. Given the high $\mathrm{S} / \mathrm{N}$ of the integrated profiles the formal uncertainties on this $\sigma_{\text {tot }}$ are very small $\left(\sim 10^{-2} \mathrm{~km} \mathrm{~s}^{-1}\right)$. As expected, when large-scale motions dominate in the integrated spectrum (i.e. high $v_{\text {shear }} / \sigma_{0}$ ratios), $\sigma_{\text {tot }}$ is significantly larger than $\sigma_{0}$, but for dispersion-dominated systems the discrepancy becomes less extreme.

\section{Discussion: influence of $\mathrm{H} \alpha$ kinematics on a galaxy's Ly $\alpha$ emission}

\subsection{Clues on Ly $\alpha$ escape mechanisms via spatially resolved $\mathrm{H} \alpha$ kinematics}

Recent theoretical modelling of the $\operatorname{Ly} \alpha$ radiative transport within realistic interstellar medium environments predicts that small-scale interstellar medium physics are a decisive factor in regulating the Ly $\alpha$ escape from galaxies (Verhamme et al. 2012; Behrens \& Braun 2014). In particular, Behrens \& Braun (2014) demonstrate how supernova blown outflow cavities become favoured escape channels for $\operatorname{Ly} \alpha$ radiation. These cavities naturally occur in zones of enhanced star formation activity, where the input of kinetic energy from supernovae and stellar winds into the surrounding interstellar medium is also expected to drive highly turbulent gas flows. Do we see supporting evidence for this scenario in our IFS observations of the LARS galaxies?

As described in Sect. 5.1, all our velocity fields are characterised by broad profiles that are best explained by turbulent flows of ionised gas. The more violent these flows are, the more likely they carve holes or bubbles through the interstellar medium through which the ionised gas eventually flows out into the galaxy's halo. Close inspection of Fig. 5 reveals, that in LARS 1, LARS 3, and LARS 13 (Fig. 7) zones of high Ly $\alpha$ surface brightness occur near or even co-spatial to zones where for these galaxies the maximum velocity dispersions are observed. In both LARS 1 and LARS 13, these zones are also co-spatial with the regions of highest $\mathrm{H} \alpha$ surface-brightness, hence, here the SFR density is highest. Moreover, in LARS 1 the $\mathrm{H} \alpha$ image shows a diffuse fan-like structure emanating outwards from this star-forming knot (Paper II; Paper I). Finally, the analysis of the COS spectra in Paper V shows blueshifted, low-ionisation state interstellar absorption lines in LARS 1 and LARS 13 indicative of outflowing neutral gas. Hence, for those two galaxies, together with the high-resolution HST imaging data, our IFS data provides further evidence for the relevance of localised outflows in shaping the observed Ly $\alpha$ morphology.

However, in LARS 3 the interpretation is already not as straightforwards. Here the broadest $\mathrm{H} \alpha$ lines do not occur cospatial with the highest SFR density. Although also here the COS spectrum shows a bulk outflow of cold neutral gas (Paper V), the locally enhanced dispersion values seen in the PMAS maps appear not to be related to this outflow. Instead, it appears more naturally that the high turbulence within the ionised gas is not primarily driven by star formation activity, but is rather the result of the violent interaction with the north-western companion (see e.g. Teyssier et al. 2010 for theoretical support). The interpretation is further complicated by the fact that the HST imaging data resolves the local Ly $\alpha$ knot on smaller scales than which we are probing with our PMAS observations.

Nevertheless, there is another example among the LARS galaxies for Ly $\alpha$ escape being related to an outflow: LARS 5 (cf. paper VI). For this galaxy, we already presumed in Paper II the existence of a starburst-driven wind based solely on the filamentary structure seen in the HST $\mathrm{H} \alpha$ image. Our dispersion map now reveals that these filaments fill the interior of a biconical structure, with its base confined on the most prominent starforming clump (see also Appendix A.5), which is fully in accordance with theoretical expectations for an evolved starburstdriven superwind (e.g. Cooper et al. 2008). Also, here Ly $\alpha$ photons could preferentially escape the disk through the cavity blown by the wind. The strongest Ly $\alpha$ enhancement is found directly above and below the main star-forming clump and the low surface brightness Ly $\alpha$ halo is then produced by those photons scattering on a bipolar shell-like structure of ambient neutral gas swept up by the superwind. Unfortunately, the brightest hot spots in Ly $\alpha$ above and below the plane are at the base of this outflowing cone and occur at scales that we cannot resolve in our PMAS data. Similar but less spectacular examples of elevated velocity dispersions above and below a disk can be seen in LARS 7 and LARS 11, with both galaxies also embedded in low surface brightness Ly $\alpha$ halos.

Another possible example for the importance of outflow kinematics in facilitating direct $\operatorname{Ly} \alpha$ escape is the most luminous LAE in the sample: LARS 14 (see also Appendix A.14). Here the observed $\mathrm{H} \alpha$ profiles are always characterised by an underlying fainter broad component (see Fig. 3) that is believed to be directly related to outflowing material (e.g. Yang et al. 2015).

We conclude that while in some individual cases a causal connection between spatially resolved $\mathrm{H} \alpha$ kinematics and localised outflow scenarios might be conjectured, globally this is not a trend seen in our observations. Furthermore, $\mathrm{H} \alpha$ kinematics tell only one part of the whole story, and the suggested outflow scenarios should also be traceable by gas with a high degree of ionisation. At least for one galaxy with $\operatorname{Ly} \alpha$ imaging similar to the LARS galaxies, such a connection has been demonstrated: ESO338-IG04. Recently, Bik et al. (2015) analysed observations of this galaxy obtained with the MUSE integral field spectrograph (Bacon et al. 2014). They show that the Ly $\alpha$ fuzz seen around the main star-forming knot of this galaxy (Hayes et al. 2005; Östlin et al. 2009) can be related to an outflow, which can be traced both with $\mathrm{H} \alpha$ kinematics and by a high degree of ionisation. In contrast to our observations, Bik et al. (2015) see the fast outflowing material in their $v_{\text {LOS }}$ map as significantly redshifted $\mathrm{H} \alpha$ emission. No similar prominent effect is apparent in our $v_{\text {LOS }}$ maps. Nevertheless, the $v_{\text {LOS }}$ fields of LARS 7 and LARS 12 display strongly localised redshifts in $\mathrm{H} \alpha$ that are spatially coincident with filamentary $\mathrm{H} \alpha$ fingers, hence, here we might also see a fast outflow pointed away from the observer. In 


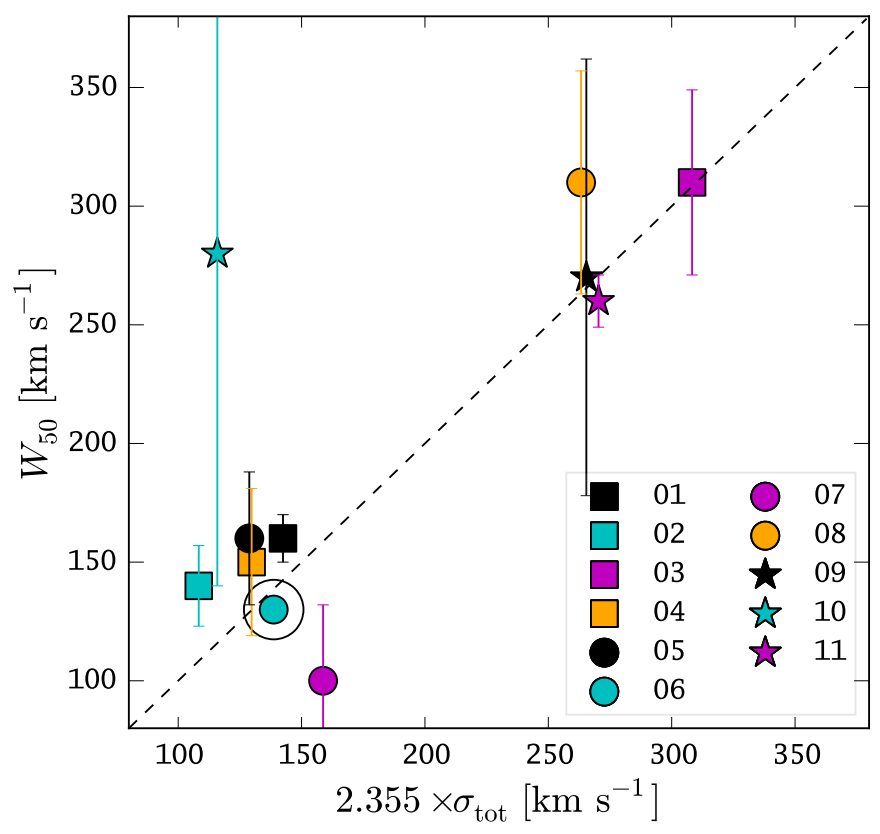

Fig. 8. Comparison of integrated $\mathrm{H}$ I linewidth $W_{50}$ to FWHM of the $\mathrm{H} \alpha$ integrated velocity dispersion $2 \sqrt{2 \ln 2} \times \sigma_{\text {tot }}$. The dashed line indicates the one-to-one relation. For LARS 6, (encircled point) we plot as $W_{50}$, a preliminary result based upon VLA C-configuration interferometry, as the beam of the GBT profile (used to derive $W_{50}$ in Paper III) is too broad to separate LARS 6 from a neighbouring galaxy.

addition, in those galaxies, no co-spatial relation between Ly $\alpha$ emissivity and the suspected outflows can be established.

\subsection{Comparison of $\mathrm{H} \alpha$ to $\mathrm{HI}$ observations}

Radiative transfer of $\operatorname{Ly} \alpha$ photons depends on the relative velocity differences between scattering $\mathrm{HI}$ atoms and Ly $\alpha$ sources. If the Ly $\alpha$ sources are out of resonance with the bulk of the neutral medium, they are less likely scattered, and in turn more likely to escape the galaxy. It is exactly this interplay between the ionised and neutral ISM phases that is determining the whole Ly $\alpha$ radiative transfer. In Paper III we studied LARS galaxies in the H I $21 \mathrm{~cm}$ line, using single-dish GBT and VLA D-configuration observations. We now attempt a comparison between the neutral and ionised gas kinematics in the LARS galaxies, cognisant of the fact that the spatial scales probed by both instruments are much larger than our PMAS H II observations.

Generally, our GBT H I spectra have low S/N, and for the three most distant LARS galaxies, LARS 12, LARS 13, and LARS 14, we could not detect any significant signal at all. The profiles are mostly single or multiple peaked, but rarely show a classical double-horn profile that would be expected for a flat rotation curve. Hence, qualitatively our observed GBT H I line profiles are consistent with our PMAS results that most of the LARS galaxies are kinematically perturbed or sometimes even strongly interacting systems.

In Paper III we measured the width of the HI lines at $50 \%$ of the line peak from the GBT spectra. In Fig. 8 we compare this quantity, $W_{50}$, to the FWHM of the integrated $\mathrm{H} \alpha$ velocity dispersion $\sigma_{\text {tot }}$ (Sect. 5.2.4). Notably, two systems deviate significantly from the one-to-one relation: LARS 7 and LARS 10. LARS 10 shows the lowest S/N H I spectrum and moreover our PMAS FoV does not capture two smaller star-forming clumps in the south-east. Therefore, we believe that observational difficulties are the source of the $\sigma_{\text {tot }}-W_{50}$ difference in this galaxy. In LARS 7, however, we suspect the difference to be genuine. In this galaxy, the $\mathrm{H} \alpha$ morphology is significantly puffed up and rounder compared to the disk-like continuum (see also Appendix A.7). Therefore, we suspect that the smaller $W_{50}$ measurement indicates that bulk of $\mathrm{HI}$ is in a kinematically more quiescent state then the ionised gas. This galaxy is one of the stronger LAEs in the sample $\left(f_{\mathrm{esc} .}^{\mathrm{Ly} \alpha}=0.14\right.$ and $E W_{\mathrm{Ly} \alpha}=40 \AA$ ). That considerable amounts of Ly $\alpha$ photons escape from LARS 7 was noted as peculiar in Paper V, since the metal absorption lines indicated there was a large amount of neutral gas sitting at the systemic velocity of the galaxy. Our observations now indicate that the intrinsic Ly $\alpha$ photons are produced in gas that is less quiescent than the scattering medium, which therefore is more transparent for a significant fraction of the intrinsic Ly $\alpha$ photons.

Spatially resolved VLA velocity fields are available for only a subset of five LARS galaxies (LARS 2, LARS 3, LARS 4, LARS 8, and LARS 9). They represent rotations disturbed by interactions with neighbours. In four of them, H I kinematical axes have similar orientations as those of the PMAS $\mathrm{H} \alpha$ velocity field. In the fifth object, the complex interacting system LARS 9, the $\mathrm{H} \alpha$ and $\mathrm{HI}$ velocity fields show different characteristics, but the complexities seen in the PMAS maps of this galaxy are on scales far beyond the spatial resolving power of the VLA D configuration (see also Appendix A.9). However, some of the LARS galaxies have already been observed with the VLA in C and B configuration and the analysis is currently in progress. For the first time, these data will allow a comparison between $\mathrm{H} \alpha, \mathrm{HI}$ and $\mathrm{Ly} \alpha$ on meaningful physical scales. It is evident that these comparisons will provide a critical benchmark for our understanding of Ly $\alpha$ radiative transfer in interstellar and circum-galactic environments.

\subsection{Relations between kinematical properties and galaxy parameters}

In Sect. 5.2 we quantified the global kinematical properties of the LARS galaxies using the non-parametric estimators $v_{\text {shear }}$, $\sigma_{0}$ and $v_{\text {shear }} / \sigma_{0}$. Before linking these observables to global Ly $\alpha$ properties of the LARS galaxies (cf. Sect. 6.4), we need to understand which galaxy parameters are encoded in them.

We find strong correlations between stellar mass $M_{\star}$ and $v_{\text {shear}}$, as well as SFR and $\sigma_{0}\left(M_{\star}\right.$ and SFR from Paper II). Graphically we show these correlations in Fig. 9 (left panel) and Fig. 10 (centre panel). The Spearman rank correlation coefficients (e.g. Wall 1996) are $\rho_{\mathrm{s}}=0.763$ for the $M_{\star}-v_{\text {shear }}$ relation and $\rho_{\mathrm{s}}=0.829$ for the SFR- $\sigma_{0}$ relation. This corresponds to likelihoods of the null hypothesis that no monotonic relation exists between the two parameters of $p_{0}=0.02 \%$ and $p_{0}=0.3 \%$ (two-tailed test ${ }^{11}$ ) for the SFR- $\sigma_{0}$ and $M_{\star}-v_{\text {shear }}$ relation, respectively. We discuss these two relations in more detail in Sect. 6.3.1 and Sect. 6.3.2, where we also introduce the comparison samples shown in Fig. 9 and Fig. 10.

Besides this tight correlation between SFR and $\sigma_{0}$, there is also a weaker correlation between SFR and $v_{\text {shear }}$ in our sample $\left(\rho_{\mathrm{s}}=0.775, p_{0}=0.1 \%\right)$. We show the data in Fig. 10 (left panel). While not shown graphically, the DYNAMO disks would scatter over the whole SFR- $v_{\text {shear }}$ plane, also filling the upper left corner in this diagram. Finally, in Fig. 10 (right panel) we

\footnotetext{
${ }^{11}$ We consider correlations as significant when the likelihood of the null hypothesis, $p_{0}$, is smaller than $5 \%$.
} 

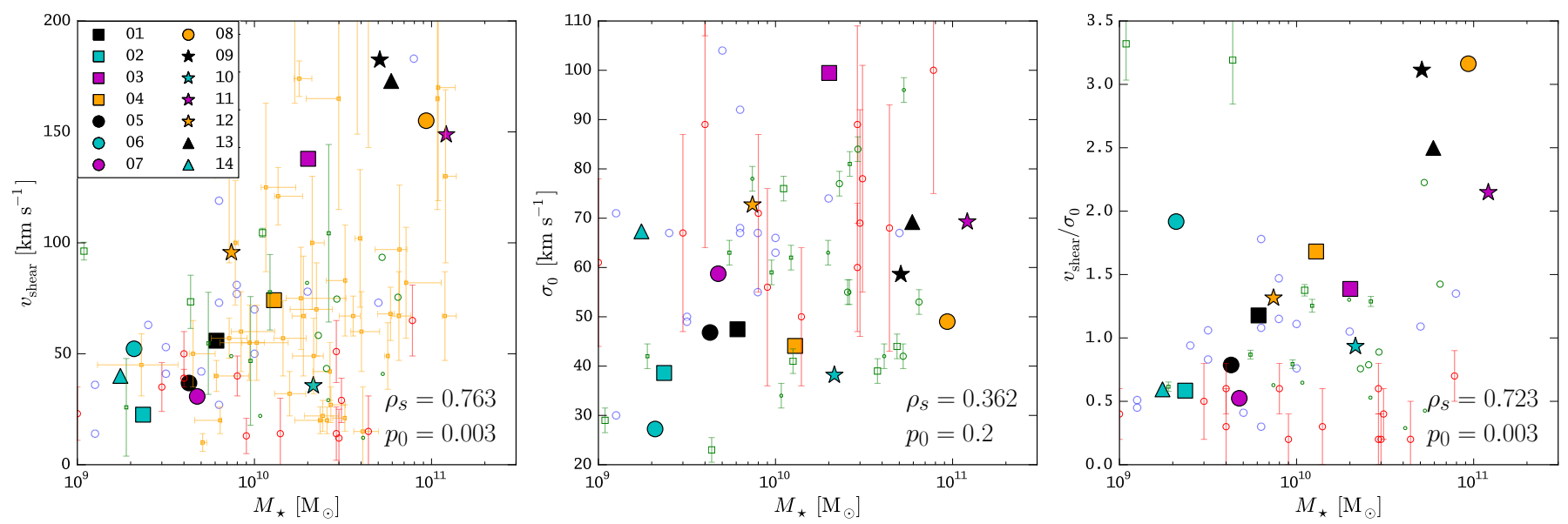

Fig. 9. Global $\mathrm{H} \alpha$ kinematical parameters $v_{\text {shear }}$ (left panel), $\sigma_{0}$ (middle panel), and $v_{\text {shear }} / \sigma_{0}$ (right panel) in comparison to stellar mass for LARS galaxies (from Paper II) and in comparison to literature values: DYNAMO $z \sim 0.1$ [compact] perturbed rotators from Green et al. (2014) as [small] green circles; DYNAMO $z \sim 0.1$ [compact] complex kinematics from Green et al. (2014) as [small] green squares; Local Lyman Break Analogues from Gonçalves et al. (2010) as blue circles; Keck/OSIRIS resolved $z \sim 2-3$ star-forming galaxies from Law et al. (2009) as red circles; SINS $z \sim 2-3$ galaxies from Förster Schreiber et al. (2009) as orange squares (only in the left panel). Uncertainties given where available. The LARS galaxies are represented by symbols according to the legend in the left panel. Spearman rank correlation coefficients $\rho_{\mathrm{s}}$ and corresponding $p_{0}$ values for the LARS galaxies are shown in each panel.
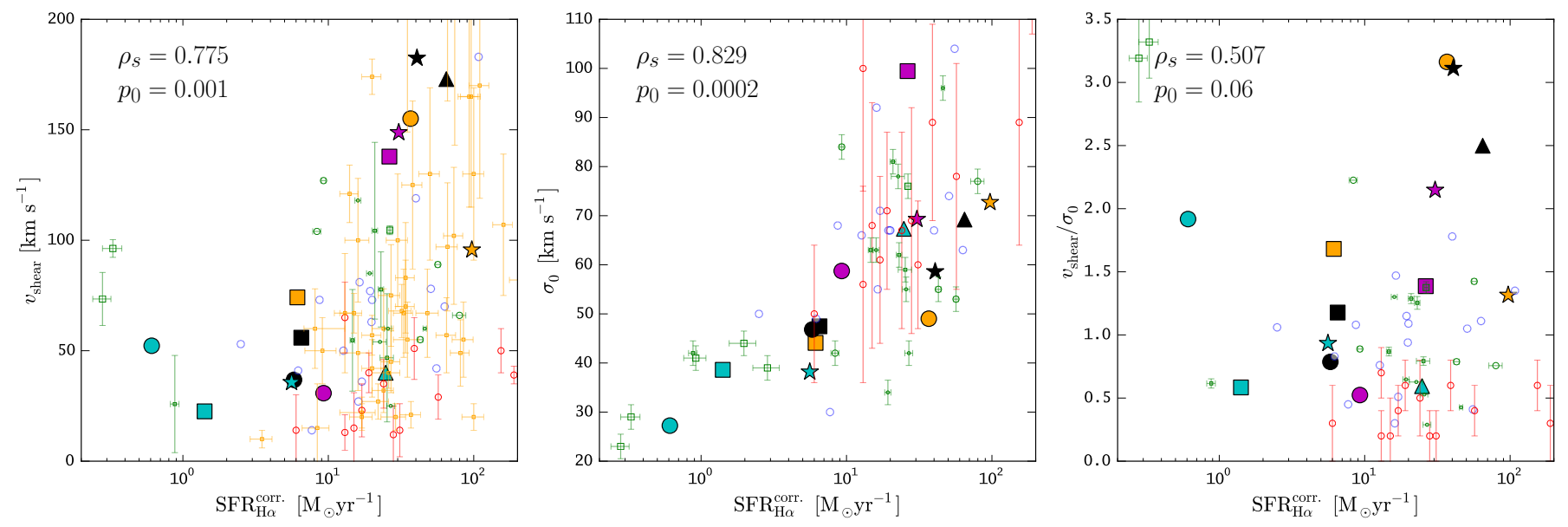

Fig. 10. Global $\mathrm{H} \alpha$ kinematical parameters $v_{\text {shear }}$ (left panel), $\sigma_{0}$ (middle panel), and $v_{\text {shear }} / \sigma_{0}$ (right panel) in comparison to SFR for LARS galaxies (from Paper II) and in comparison to literature values (same symbols as in Fig. 9). Uncertainties given where available. Spearman rank correlation coefficients $\rho_{\mathrm{s}}$ and corresponding $p_{0}$ values for the LARS galaxies are shown in each panel.

find that $v_{\text {shear }} / \sigma_{0}$ is not significantly correlated with the SFR in LARS $\left(\rho_{\mathrm{s}}=0.507, p_{0}=6 \%\right)$.

We also check in Fig. 9 (centre panel) for a $M_{\star}-\sigma_{0}$ correlation, but with $\rho_{\mathrm{s}}=0.362$ the null hypothesis that the variables are uncorrelated cannot be rejected $\left(p_{0}=20 \%\right)$. Similar low correlation-coefficients are found for the comparison samples in Fig. 9 (centre panel). Since there is likely a monotonic relation between $M_{\star}-v_{\text {shear }}$ and $M_{\star}-\sigma_{0}$ are uncorrelated, the monotonic relation $M_{\star}-v_{\text {shear }} / \sigma_{0}\left(\rho_{\mathrm{s}}=0.723, p_{0}=0.3 \%\right)-$ Fig. 9 (right panel) - is expected.

From these results, we conclude that dispersion-dominated galaxies in our sample are preferentially low-mass systems with $M_{\star} \lesssim 10^{10} M_{\odot}$. This result is also commonly found in samples of high- $z$, star-forming galaxies (Law et al. 2009; Förster Schreiber et al. 2009; Newman et al. 2013).

\subsubsection{SFR- $\sigma_{0}$ correlation}

The highly significant correlation between $\sigma_{0}$ and SFR has long been established for giant H II regions (Terlevich \& Melnick 1981). Recently, it has been shown that it extends over a large dynamical range in star formation and mass, not only locally but also at high redshifts (Green et al. 2010, 2014). The physical nature of the $\sigma_{0}$-SFR relation might be that star formation feedback powers turbulence in the interstellar medium. On the other hand, the processes that lead to a high SFR might also be responsible for producing a high $\sigma_{0}$. In particular, inflows of cold gas that feed the star formation processes are expected to stir up the interstellar medium and thus lead to turbulent flows (e.g. Wisnioski et al. 2015, and references therein).

Graphically we compare, in Fig. 10 (centre panel), the LARS SFR- $\sigma_{0}$ points to the values from the DYNAMO 
galaxies (Green et al. 2014) with complex kinematics and the DYNAMO perturbed rotators. To put this result in context with high- $z$ studies, in Fig. 10 we also show the SFR- $\sigma_{0}$ points from the Keck/OSIRIS $z \sim 2-3$ star-forming galaxies by Law et al. (2009), and the local Lyman break analogues by Gonçalves et al. (2010). An exhaustive compilation of SFR- $\sigma_{0}$ measurements is presented in Green et al. (2014), and the LARS SFR- $\sigma_{0}$ points do not deviate from this relation.

\subsection{2. $M_{\star}-v_{\text {shear }}$ correlation}

Given the complexity of the ionised gas velocity fields seen in the LARS galaxies, a tight relation between our measured, inclination-uncorrected $v_{\text {shear }}$ values with stellar mass appears surprising. It indicates that, at least in a statistical sense, $v_{\text {shear }}$ is tracing systemic rotation in our systems and that the scatter in our relation is dominated by the unknown inclination correction (see also Law et al. 2009).

To put our data in context with other studies, we compare our $M_{\star}-v_{\text {shear }}$ data points in Fig. 9 (left panel) to the Green et al. (2014) DYNAMO sample and to the Gonçalves et al. (2010) local Lyman break analogues. Our high- $z$ comparison samples are the Keck/OSIRIS $z \sim 2-3$ star-forming galaxies Law et al. and the $z \sim 1-3$ SINS sample by Förster Schreiber et al. (2009). For the rotation-dominated and perturbed rotators in the DYNAMO sample, Green et al. tabulate rotation velocity at 2.2 disk scale lengths obtained from fitting model disks to their velocity fields. We convert these to an inclination-uncorrected value via multiplication with $\sin i$. Again, for the DYNAMO sample we only compare to their objects with complex kinematics or their perturbed rotators, as they are dominant in our sample.

It is apparent from Fig. 9 (left panel) that while our data points line up well with the $z \sim 0.1$ galaxies, a significant number of high- $z$ galaxies shows lower $v_{\text {shear }}$ values at given stellar mass in the range $10^{10} M_{\odot}-10^{11} M_{\odot}$. The reason for this is that high- $z$ studies are not sensitive enough to reach the outer faint isophotes, hence, they are biased towards lower $v_{\text {shear }}$ values (see Sect. 5.2.1). Indeed, Law et al. (2009) report a detection limit of $\sim 1 M_{\odot} \mathrm{yr}^{-1} \mathrm{kpc}^{-2}$, while Förster Schreiber et al. (2009) report $\approx 0.03 M_{\odot} \mathrm{yr}^{-1} \mathrm{kpc}^{-2}$ as an average for their sample, which is comparable to our depth (Sect. 5.1).

\subsection{Relations between global kinematical properties and Ly $\alpha$ observables}

We now explore trends between global kinematical properties derived from the ionised gas in the LARS galaxies and their Ly $\alpha$ observables determined in Paper II, namely Ly $\alpha$ escape fraction $f_{\mathrm{esc}}^{\mathrm{Ly} \alpha}$, ratio of $\mathrm{Ly} \alpha$ to $\mathrm{H} \alpha$ flux $\mathrm{Ly} \alpha / \mathrm{H} \alpha$, and $\mathrm{Ly} \alpha$ equivalent width $E W_{\mathrm{Ly} \alpha}$. For reference, we list these quantities here again in Table 2 . We recall that $\mathrm{Ly} \alpha / \mathrm{H} \alpha$ is the observed flux ratio, while $f_{\text {esc }}$ is determined from the intrinsic luminosities, i.e. after correcting the fluxes for dust reddening.

Regarding the qualitative classification of the $v_{\text {LOS }}$ maps in Sect. 5.1 into rotating disks, perturbed rotators and objects showing complex kinematics there is no preference in any of the globally integrated Ly $\alpha$ observables. Both the maximum and minimum of these observables occur in the complex kinematics class. Therefore, just the qualitative appearance of the velocity field seems not to predict whether the galaxy is a LAE or not.

In the previous section, we showed that within the LARS sample higher SFRs are found in galaxies that have higher $\sigma_{0}$ and higher $v_{\text {shear }}$ measurements and that galaxies of higher mass also show higher $v_{\text {shear }}$ values and higher $v_{\text {shear }} / \sigma_{0}$ ratios (Figs. 9 and 10). We now compare our $v_{\text {shear }}, \sigma_{0}$ and $v_{\text {shear }} / \sigma_{0}$-ratios to the aperture integrated Ly $\alpha$ observables $E W_{\mathrm{Ly} \alpha}, \mathrm{Ly} \alpha / \mathrm{H} \alpha$ and $f_{\text {esc }}^{\mathrm{Ly} \alpha}$ from Paper II. We do this in form of a graphical $3 \times 3$ matrix in Fig. 11. In each panel, we include the Spearman rank correlation coefficient $\rho_{\mathrm{s}}$ and the likelihood $p_{0}$ to reject the null hypothesis.

From the centre row in Fig. 11 it is obvious that none of the Ly $\alpha$ observables correlates with the averaged intrinsic velocity dispersion $\sigma_{0}$. As $\sigma_{0}$ correlates positively with SFR, this signifies that the observed Ly $\alpha$ emission is a bad SFR calibrator. In Fig. 11 it is also evident that galaxies with higher shearing velocities $\left(v_{\text {shear }} \gtrsim 50 \mathrm{~km} \mathrm{~s}^{-1}\right)$ have preferentially lower $E W_{\mathrm{Ly} \alpha}$, lower Ly $\alpha / \mathrm{H} \alpha$, and lower $f_{\mathrm{esc}}^{\mathrm{Ly} \alpha}$. Therefore, according to the above presented $M_{\star}-v_{\text {shear }}$ relation (Sect. 6.3.2) LAEs are preferentially found among the systems with $M_{\star} \lesssim 10^{10} M_{\odot}$ in LARS. This deficiency of strong LAEs among high-mass systems was already noted in Paper II. Here we see this trend from a kinematical perspective now. Also, a low $v_{\text {shear }}$ value, although a necessary condition, seems not to be sufficient to have significant amounts Ly $\alpha$ photons escaping (e.g. LARS 6). Again, this shows that Ly $\alpha$ escape from galaxies is a complex multi-parametric problem.

Although the LARS sample is small, the result that seven of eight non-LAEs have $v_{\text {shear }} / \sigma_{0}>1$ and four of six LAEs have $v_{\text {shear }} / \sigma_{0}<1$ signals that dispersion-dominated kinematics are an important ingredient in Ly $\alpha$ escape. Again, lower $v_{\text {shear }} / \sigma_{0}$ ratios are found in lower $M_{\star}$ objects in our sample and $\sigma_{0}$ is uncorrelated with $M_{\star}$. Therefore, the correlation between Ly $\alpha$ observables and $v_{\text {shear }} / \sigma_{0}$ is a consequence of the correlation between Ly $\alpha$ observables and $M_{\star}$. Nevertheless, low $v_{\text {shear }} / \sigma_{0}$ ratios also state that the ionised gas in those galaxies must be in a turbulent state. High SFR appears to be connected to an increase turbulence, but it is currently not clear whether the processes that cause star formation or feedback from star formation are responsible for the increased turbulence (Sect. 6.3.1). Regardless of the causal relationship between SFR and $\sigma_{0}$, our results support that $\operatorname{Ly} \alpha$ escape is being favoured in low-mass systems undergoing an intense star formation episode. Similarly Cowie et al. (2010) found that in a sample of UV bright $z \sim 0.3$ galaxies, LAEs are primarily the young galaxies that have recently become strongly star forming. In the local Universe dispersiondominated systems with $v_{\text {shear }} / \sigma_{0}<1$ are rare, but they become much more prevalent at higher redshifts (Wisnioski et al. 2015). Coincidentally, the number density of LAEs rises towards higher redshifts (Wold et al. 2014). Therefore we speculate, that dispersion-dominated kinematics are indeed a necessary requirement for a galaxy to have a significant amount of Ly $\alpha$ photons escaping.

\subsection{Ly $\alpha$ extension and $\mathrm{H} \alpha$ kinematics}

All of the LARS LAEs show a significantly more extended Ly $\alpha$ morphology compared to their appearance in $\mathrm{H} \alpha$ or UV continuum. These large-scale Ly $\alpha$ haloes appear to completely encompass the star-forming regions. LARS 1, LARS 2, LARS 5, LARS 7, LARS 12 and LARS 14 are the most obvious examples of extended Ly $\alpha$ emission, but the phenomenon is visible in all our objects, even for those galaxies that show Ly $\alpha$ in absorption (Figs. 5-7; cf. Hayes et al. 2013 and Paper II). At high redshift the ubiquity of extended Ly $\alpha$ haloes around LAEs was recently revealed by Wisotzki et al. (2015) on an individual object-by-object basis. However, in contrast to the LARS 
E. Ch. Herenz et al.: LARS. Spatially resolved $\mathrm{H} \alpha$ kinematics
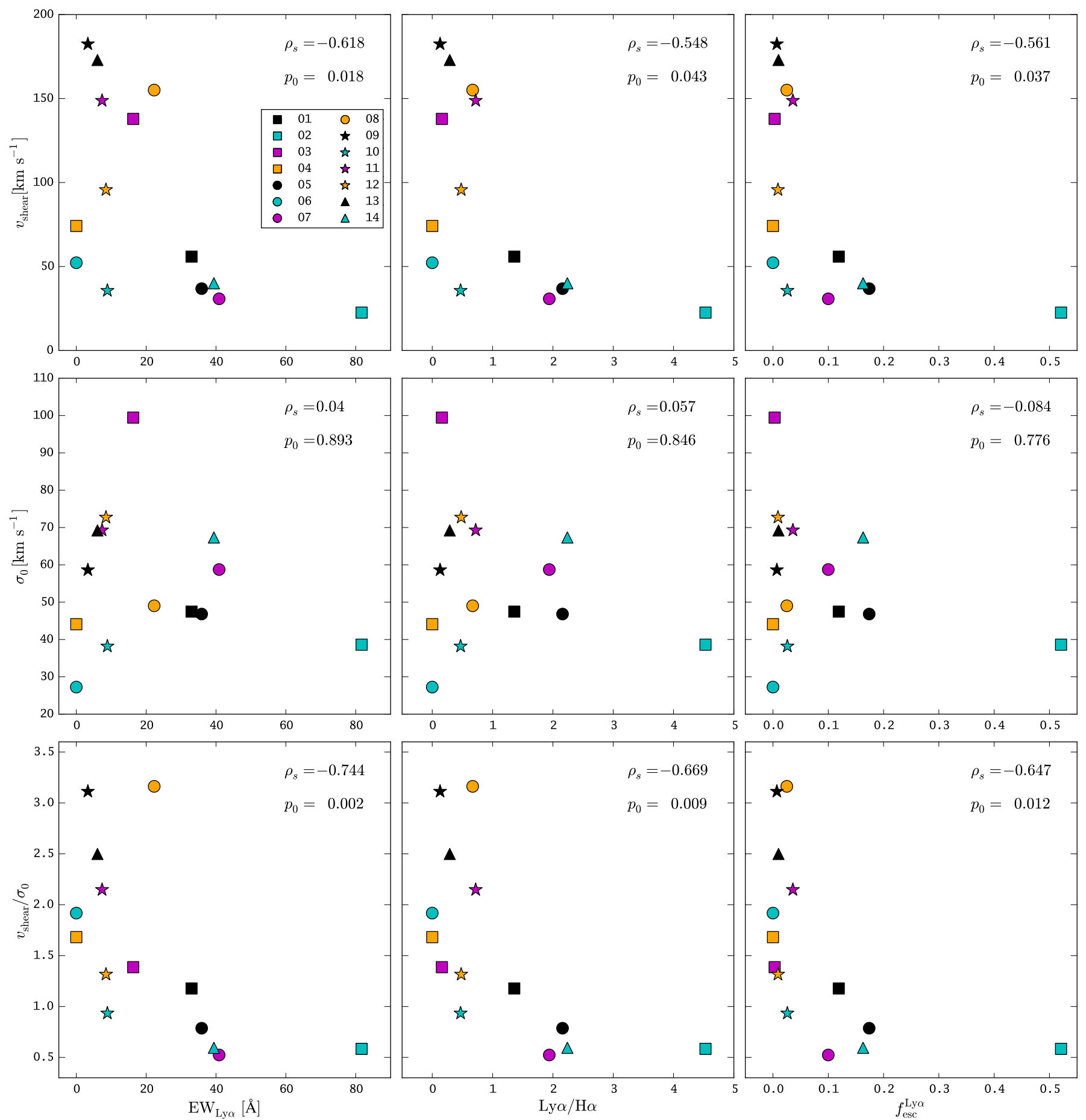

Fig. 11. Relations between global Ly $\alpha$ properties of the LARS galaxies to global kinematical parameters $v_{\text {shear }}, \sigma_{0}$ and $v_{\text {shear }} / \sigma_{0}$.

Ly $\alpha$ haloes, the high- $z$ haloes appear to have $\sim 10 \times$ larger extents at a given continuum radius (Wisotzki et al. 2015, their Fig. 12). In order to quantify the spatial extent of observed relative to intrinsic Ly $\alpha$ emission in Hayes et al. (2013) we defined the "Relative Petrosian Extension" $\xi_{\mathrm{Ly} \alpha}$ as the ratio of the Petrosian radii (Petrosian 1976) at $\eta=20 \%$ measured in Ly $\alpha$ and $\mathrm{H} \alpha: \xi_{\mathrm{Ly} \alpha}=R_{\mathrm{P} 20}^{\mathrm{Ly} \alpha} / R_{\mathrm{P} 20}^{\mathrm{H} \alpha}$. For reference, we list the $\xi_{\mathrm{Ly} \alpha}$ values of the LARS galaxies here again in Table 2. Based on an anti-correlation between $\xi_{\mathrm{Ly} \alpha}$ and UV slope we conjectured that smaller Ly $\alpha$ haloes occur in galaxies that have converted more of their circum-galactic neutral gas into forming stars and subsequently dust (Hayes et al. 2013). In such a scenario, the larger extent of high- $z$ Ly $\alpha$ haloes could be related to the circum-galactic gas-reservoirs being larger in the early universe.

In Fig. 12 we show that there are no significant correlations between $\xi_{\text {Ly } \alpha}$ and the global kinematical $\mathrm{H} \alpha$ parameters $v_{\text {shear }}$, $\sigma_{0}$ and $v_{\text {shear }} / \sigma_{0}$. Therefore, we reason that the kinematics of the interstellar medium do not strongly influence the appearance of the haloes and that the $\operatorname{Ly} \alpha$ halo phenomenon is only related to the presence of a full gas reservoir. Further, $21 \mathrm{~cm} \mathrm{HI} \mathrm{imaging}$ 

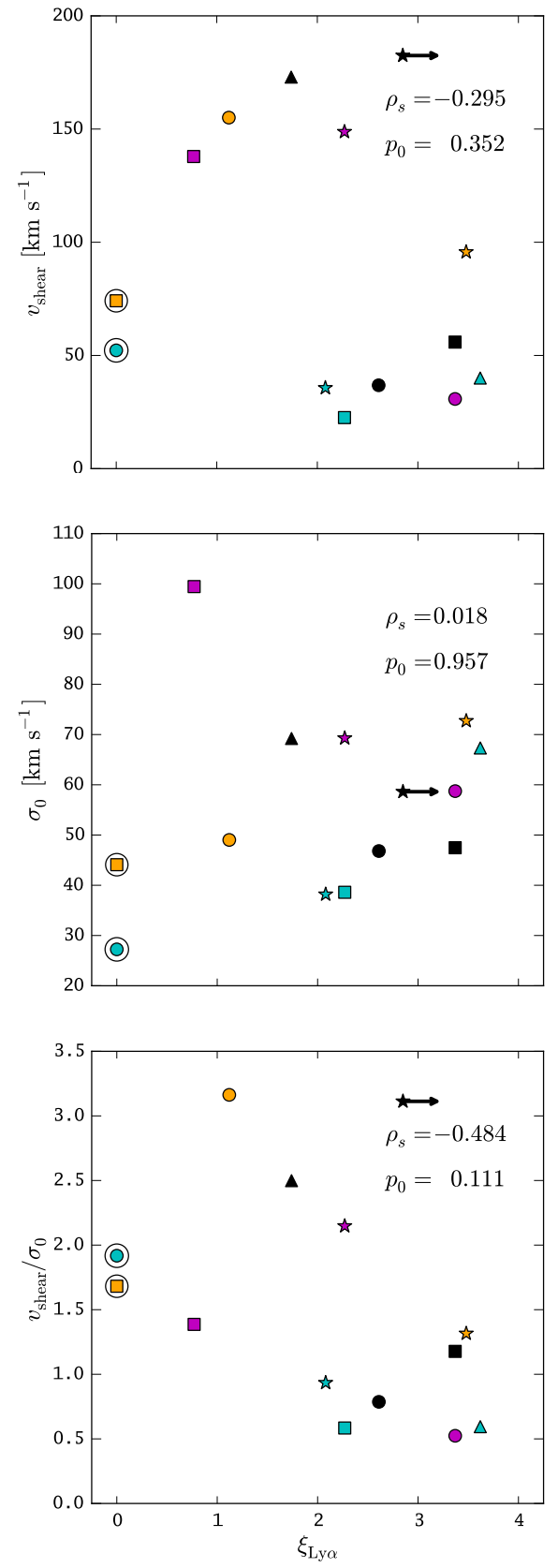

Fig. 12. Relations between $\xi_{\text {Ly } \alpha}$, the "relative Petrosian extension of Ly $\alpha$ " as defined in Hayes et al. (2013), and global kinematical parameters $v_{\text {shear }}, \sigma_{0}$ and $v_{\text {shear }} / \sigma_{0}$ with symbols according to the legend in Fig. 9. For galaxies with no Ly $\alpha$ emission (LARS 4 and LARS 6, circled symbols) $\xi_{\mathrm{Ly} \alpha}$ is defined as zero and for LARS 9 the measured $\xi_{\text {Ly } \alpha}$ presents a lower limit (see also Hayes et al. 2013). Spearman rank correlation coefficients $\rho_{\mathrm{s}}$ and corresponding $p_{0}$ values are calculated excluding LARS 4 and LARS 6.

of the LARS galaxies at high spatial resolution is needed to test this scenario.

\section{Summary and conclusions}

We obtained the following results from our integral field spectroscopic observations of the $\mathrm{H} \alpha$ line in the LARS galaxies:

1. Half of the LARS galaxies show complex $\mathrm{H} \alpha$ kinematics. There are kinematical properties consistent with a rotating disk in only two galaxies and in five a disturbed rotational signature is apparent. With respect to Ly $\alpha$ escape, we find no preference of high $\mathrm{EW}_{\mathrm{Ly} \alpha}$ or high $f_{\mathrm{esc}}^{\mathrm{Ly} \alpha}$. values towards any of those classes, but the minimum and maximum values both occur in objects showing complex kinematics in our sample.

2. A common feature in all LARS galaxies are high $\mathrm{H} \alpha$ velocity dispersions. With $v_{\text {FWHM }} \gtrsim 100 \mathrm{~km} \mathrm{~s}^{-1}$ our measurements are in contrast to values typically seen in local spirals, but in high- $z$ star-forming galaxies such high values appear to be the norm.

3. While we could not infer a direct relation between spatially resolved kinematics of the ionised gas and photometric Ly $\alpha$ properties for all LARS galaxies, in individual cases our maps appear qualitatively consistent with outflow scenarios that promote Ly $\alpha$ escape from high-density regions.

4. Currently, a spatially resolved comparison of our H II velocity fields to our H I data is severely limited since the scales resolved by the radio observations are significantly larger. However, for one galaxy (LARS 7) the difference between globally integrated velocity dispersion of ionised and neutral gas offers a viable explanation for the escape of significant amounts of Ly $\alpha$ photons.

5. From our $\mathrm{H} \alpha$ velocity maps, we derive the non-parametric statistics $v_{\text {shear }}, \sigma_{0}$ and $v_{\text {shear }} / \sigma_{0}$ to quantify the kinematics of the LARS galaxies globally. Our $v_{\text {shear values range from }}$ $30 \mathrm{~km} \mathrm{~s}^{-1}$ to $180 \mathrm{~km} \mathrm{~s}^{-1}$ and our $\sigma_{0}$ values range from $40 \mathrm{~km} \mathrm{~s}^{-1}$ to $100 \mathrm{~km} \mathrm{~s}^{-1}$. For our ratios $v_{\text {shear }} / \sigma_{0}$, we find a median of 1.4, and five of the LARS galaxies are dispersiondominated systems with $v_{\text {shear }} / \sigma_{0}<1$.

6. A positively correlated $\sigma_{0}$ with SFR in the LARS galaxies is fully consistent with other IFS studies. We also find strong correlations between $M_{\star}$ and $v_{\text {shear }}, M_{\star}$ and $v_{\text {shear }} / \sigma_{0}$, and SFR and $v_{\text {shear }}$. In view of the $M_{\star}-v_{\text {shear }}$ correlation the SFR$v_{\text {shear }}$ correlation implies that more massive galaxies have higher overall star formation rates in our sample.

7. The $\mathrm{Ly} \alpha$ properties $\mathrm{EW}_{\mathrm{Ly} \alpha}, \mathrm{Ly} \alpha / \mathrm{H} \alpha$ and $f_{\mathrm{esc}}^{\mathrm{Ly} \alpha}$ do not correlate with $\sigma_{0}$, but they correlate with $v_{\text {shear }}$ and $v_{\text {shear }} / \sigma_{0}$ (Fig. 11). We find no correlation between the global kinematical statistics and the extent of the Ly $\alpha$ halo.

8. Four of six LARS LAEs are dispersion-dominated systems with $v_{\text {shear }} / \sigma_{0}<1$ and 7 of 8 non-LAEs have $v_{\text {shear }} / \sigma_{0}>1$.

Observational studies of $\operatorname{Ly} \alpha$ emission in local-Universe galaxies have so far focused on imaging and UV spectroscopy (for a recent review see Hayes 2015). For the first time, we provided empirical results from IFS observations for a sample of galaxies with known Ly $\alpha$ observables. In our pioneering study, we focused on the spectral and spatial properties of the intrinsic $\operatorname{Ly} \alpha$ radiation field as traced by $\mathrm{H} \alpha$. We found a direct relation between the global non-parametric kinematical statistics of the ionised gas and the $\operatorname{Ly} \alpha$ observables $f_{\mathrm{esc}}^{\mathrm{Ly} \alpha}$ and $E W_{\mathrm{Ly} \alpha}$, and our main result is that dispersion-dominated systems favour Ly $\alpha$ escape. The prevalence of LAEs among dispersion-dominated galaxies could be simply a consequence of these systems being the lower mass systems in our sample, which is a result already found in Paper II. However, the observed turbulence in actively star-forming systems should be related to ISM conditions that ease Ly $\alpha$ radiative transfer out of high-density environments. In particular, if turbulence is a direct consequence of star formation, then the energetic input from the star formation episode might also be powerful enough to drive cavities through the neutral medium into the lower density circum-galactic environments. Of course, the kinematics of the ionised gas offer only a limited view of the processes at play, and in future studies 
we will attempt to connect our kinematic measurements to spatial mappings of the ISMs ionisation state. The synergy between IFS data, space-based UV imaging, and high-resolution $\mathrm{H} \mathrm{I} \mathrm{ob-}$ servations of nearby star-forming galaxies will be vital to build a coherent observational picture of $\operatorname{Ly} \alpha$ radiative transport in galaxies.

Acknowledgements. E.C.H. especially thanks Sebastian Kamann and Bernd Husemann for teaching him how to operate the PMAS instrument. We thank the support staff at Calar Alto observatory for help with the visitor-mode observations. All plots in this paper were created using matplotlib (Hunter 2007). Intensity related images use the cubhelix colour scheme by Green (2011). This research made extensive use of the astropy pacakge (Astropy Collaboration et al. 2013). M.H. acknowledges the support of the Swedish Research Council, Vetenskapsrådet, and the Swedish National Space Board (SNSB) and is Academy Fellow of the Knut and Alice Wallenberg Foundation. HOF is currently granted by a Cátedra CONACyT para Jóvenes Investigadores. I.O. has been supported by the Czech Science Foundation grant GACR 14-20666P. D.K. is funded by the Centre National d'Études Spatiales (CNES). F.D. is grateful for financial support from the Japan Society for the Promotion of Science (JSPS) fund. P.L. acknowledges support from the ERCStG grant EGGS-278202.

\section{References}

Adams, J. J., Blanc, G. A., Hill, G. J., et al. 2011, ApJS, 192, 5 Ahn, S.-H., Lee, H.-W., \& Lee, H. M. 2003, MNRAS, 340, 863

Alonso-Herrero, A., García-Marín, M., Monreal-Ibero, A., et al. 2009, A\&A, 506,1541

Astropy Collaboration, Robitaille, T. P., Tollerud, E. J., et al. 2013, A\&A, 558, A33

Bacon, R., Vernet, J., Borisiva, E., et al. 2014, The Messenger, 157, 13

Bacon, R., Brinchmann, J., Richard, J., et al. 2015, A\&A, 575, A75

Barnes, L. A., Haehnelt, M. G., Tescari, E., \& Viel, M. 2011, MNRAS, 416, 1723

Bassett, R., Glazebrook, K., Fisher, D. B., et al. 2014, MNRAS, 442, 3206

Basu-Zych, A. R., Gonçalves, T. S., Overzier, R., et al. 2009, ApJ, 699, L118

Behrens, C., \& Braun, H. 2014, A\&A, 572, A74

Behrens, C., Dijkstra, M., \& Niemeyer, J. C. 2014, A\&A, 563, A77

Bettoni, D., Galletta, G., Rampazzo, R., et al. 2011, A\&A, 534, A24

Bik, A., Östlin, G., Hayes, M., et al. 2015, A\&A, 576, L13

Calabretta, M. R., \& Greisen, E. W. 2002, A\&A, 395, 1077

Cannon, J. M., Skillman, E. D., Kunth, D., et al. 2004, ApJ, 608, 768

Cappellari, M., \& Copin, Y. 2003, MNRAS, 342, 345

Cardamone, C., Schawinski, K., Sarzi, M., et al. 2009, MNRAS, 399, 1191

Cassata, P., Le Fèvre, O., Garilli, B., et al. 2011, A\&A, 525, A143

Cassata, P., Tasca, L. A. M., Le Fèvre, O., et al. 2015, A\&A, 573, A24

Chonis, T. S., Blanc, G. A., Hill, G. J., et al. 2013, ApJ, 775, 99

Christensen, L., Laursen, P., Richard, J., et al. 2012, MNRAS, 427, 1973

Ciardullo, R., Gronwall, C., Wolf, C., et al. 2012, ApJ, 744, 110

Cooper, J. L., Bicknell, G. V., Sutherland, R. S., \& Bland-Hawthorn, J. 2008, ApJ, 674, 157

Cowie, L. L., Barger, A. J., \& Hu, E. M. 2010, ApJ, 711, 928

Cox, A. N. 2000, Sky Telesc., 100, 72

Davies, R., Förster Schreiber, N. M., Cresci, G., et al. 2011, ApJ, 741, 69

Diehl, S., \& Statler, T. S. 2006, MNRAS, 368, 497

Dijkstra, M. 2014, PASA, 31, 40

Dijkstra, M., Haiman, Z. \& Spaans, M. 2006, ApJ, 649, 14

Duval, F., Schaerer, D., Östlin, G., \& Laursen, P. 2014, A\&A, 562, A52

Duval, F., Ostlin, G., Hayes, M., et al. 2016, A\&A, 587, A77 (Paper VI)

Epinat, B., Amram, P., \& Marcelin, M. 2008a, MNRAS, 390, 466

Epinat, B., Amram, P., Marcelin, M., et al. 2008b, MNRAS, 388, 500

Epinat, B., Amram, P., Balkowski, C., \& Marcelin, M. 2010, MNRAS, 401, 2113

Erb, D. K., Steidel, C. C., Trainor, R. F., et al. 2014, ApJ, 795, 33

Erroz-Ferrer, S., Knapen, J. H., Leaman, R., et al. 2015, MNRAS, 451, 1004

Finkelstein, S. L., Papovich, C., Dickinson, M., et al. 2013, Nature, 502, 524

Flores, H., Hammer, F., Puech, M., Amram, P., \& Balkowski, C. 2006, A\&A 455,107

Förster Schreiber, N. M., Genzel, R., Bouché, N., et al. 2009, ApJ, 706, 1364

Genzel, R., Tacconi, L. J., Eisenhauer, F., et al. 2006, Nature, 442, 786

Glazebrook, K. 2013, PASA, 30, 56

Gonçalves, T. S., Basu-Zych, A., Overzier, R., et al. 2010, ApJ, 724, 1373

Green, D. A. 2011, Bull. Astron. Soc. India, 39, 289
Green, A. W., Glazebrook, K., McGregor, P. J., et al. 2010, Nature, 467, 684 Green, A. W., Glazebrook, K., McGregor, P. J., et al. 2014, MNRAS, 437, 1070 Greisen, E. W., \& Calabretta, M. R. 2002, A\&A, 395, 1061

Gronke, M., \& Dijkstra, M. 2014, MNRAS, 444, 1095

Gronke, M., Bull, P., \& Dijkstra, M. 2015, ApJ, 812, 123

Gronwall, C., Ciardullo, R., Hickey, T., et al. 2007, ApJ, 667, 79

Grove, L. F., Fynbo, J. P. U., Ledoux, C., et al. 2009, A\&A, 497, 689

Guaita, L., Francke, H., Gawiser, E., et al. 2013, A\&A, 551, A93

Guaita, L., Melinder, J., Hayes, M., et al. 2015, A\&A, 576, A51 (Paper IV)

Hashimoto, T., Ouchi, M., Shimasaku, K., et al. 2013, ApJ, 765, 70

Hashimoto, T., Verhamme, A., Ouchi, M., et al. 2015, ApJ, 812, 157

Hayes, M. 2015, PASA, 32, 27

Hayes, M., Östlin, G., Mas-Hesse, J. M., et al. 2005, A\&A, 438, 71

Hayes, M., Östlin, G., Mas-Hesse, J. M., \& Kunth, D. 2009, AJ, 138, 911

Hayes, M., Östlin, G., Schaerer, D., et al. 2010, Nature, 464, 562

Hayes, M., Östlin, G., Schaerer, D., et al. 2013, ApJ, 765, L27

Hayes, M., Östlin, G., Duval, F., et al. 2014, ApJ, 782, 6 (Paper II)

Henry, A., Scarlata, C., Martin, C. L., \& Erb, D. 2015, ApJ, 809, 19

Hong, S., Dey, A., \& Prescott, M. K. M. 2014, PASP, 126, 1048

Horne, K. 1986, PASP, 98, 609

Hu, E. M., Cowie, L. L., \& McMahon, R. G. 1998, ApJ, 502, L99

Hunter, J. D. 2007, Comput. Sci. Eng., 9, 90

Husemann, B., Kamann, S., Sandin, C., et al. 2012, A\&A, 545, A137

Husemann, B., Jahnke, K., Sánchez, S. F., et al. 2013, A\&A, 549, A87

Iye, M. 2011, Proc. Jap. Acad. Ser. B, 87, 575

Jaskot, A. E., \& Oey, M. S. 2014, ApJ, 791, L19

Jiménez-Vicente, J., Battaner, E., Rozas, M., Castañeda, H., \& Porcel, C. 1999 A\&A, 342, 417

Kennicutt, Jr., R. C. 1998, ARA\&A, 36, 189

Kunth, D., Mas-Hesse, J. M., Terlevich, E., et al. 1998, A\&A, 334, 11

Landman, D. A., Roussel-Dupre, R., \& Tanigawa, G. 1982, ApJ, 261, 732

Laursen, P., \& Sommer-Larsen, J. 2007, ApJ, 657, L69

Laursen, P., Sommer-Larsen, J., \& Andersen, A. C. 2009, ApJ, 704, 1640

Laursen, P., Duval, F., \& Östlin, G. 2013, ApJ, 766, 124

Law, D. R., Steidel, C. C., Erb, D. K., et al. 2009, ApJ, 697, 2057

Lenz, D. D., \& Ayres, T. R. 1992, PASP, 104, 1104

Lupton, R., Blanton, M. R., Fekete, G., et al. 2004, PASP, 116, 133

Malhotra, S., \& Rhoads, J. E. 2004, ApJ, 617, L5

Mallery, R. P., Mobasher, B., Capak, P., et al. 2012, ApJ, 760, 128

Marino, A., Iodice, E., Tantalo, R., et al. 2009, A\&A, 508, 1235

Martin, D. C., Chang, D., Matuszewski, M., et al. 2014, ApJ, 786, 106

Mas-Hesse, J. M., Kunth, D., Tenorio-Tagle, G., et al. 2003, ApJ, 598, 858

McLinden, E. M., Finkelstein, S. L., Rhoads, J. E., et al. 2011, ApJ, 730, 136

McLinden, E. M., Rhoads, J. E., Malhotra, S., et al. 2014, MNRAS, 439, 446

Monreal-Ibero, A., Arribas, S., Colina, L., et al. 2010, A\&A, 517, A28

Neufeld, D. A. 1990, ApJ, 350, 216

Newman, S. F., Genzel, R., Förster Schreiber, N. M., et al. 2013, ApJ, 767, 104

Oesch, P. A., van Dokkum, P. G., Illingworth, G. D., et al. 2015, ApJ, 804, L30

Oke, J. B. 1990, AJ, 99, 1621

Ono, Y., Ouchi, M., Mobasher, B., et al. 2012, ApJ, 744, 83

Östlin, G., Amram, P., Masegosa, J., Bergvall, N., \& Boulesteix, J. 1999, A\&AS, 137,419

Östlin, G., Amram, P., Bergvall, N., et al. 2001, A\&A, 374, 800

Östlin, G., Hayes, M., Kunth, D., et al. 2009, AJ, 138, 923

Östlin, G., Hayes, M., Duval, F., et al. 2014, ApJ, 797, 11 (Paper I)

Ouchi, M., Shimasaku, K., Akiyama, M., et al. 2008, ApJS, 176, 301

Pardy, S. A., Cannon, J. M., Östlin, G., et al. 2014, ApJ, 794, 101 (Paper III)

Partridge, R. B., \& Peebles, P. J. E. 1967, ApJ, 147, 868

Petrosian, V. 1976, ApJ, 209, L1

Puech, M., Hammer, F., Flores, H., Östlin, G., \& Marquart, T. 2006, A\&A, 455, 119

Rauch, M., Haehnelt, M., Bunker, A., et al. 2008, ApJ, 681, 856

Rhoads, J. E., Malhotra, S., Richardson, M. L. A., et al. 2014, ApJ, 780, 20

Rivera-Thorsen, T. E., Hayes, M., Östlin, G., et al. 2015, ApJ, 805, 14 (Paper V)

Robertson, J. G. 2013, PASA, 30, 48

Roth, M. M., Kelz, A., Fechner, T., et al. 2005, PASP, 117, 620

Roth, M. M., Fechner, T., Wolter, D., et al. 2010, in High Energy, Optical, and Infrared Detectors for Astronomy IV, SPIE Conf. Ser., 7742, 774209

Sánchez, S. F., Aceituno, J., Thiele, U., Pérez-Ramírez, D., \& Alves, J. 2007a, PASP, 119, 1186

Sánchez, S. F., Cardiel, N., Verheijen, M. A. W., et al. 2007b, A\&A, 465, 207

Sánchez, S. F., Kennicutt, R. C., Gil de Paz, A., et al. 2012, A\&A, 538, A8

Sandberg, A., Östlin, G., Hayes, M., et al. 2013, A\&A, 552, A95

Sandberg, A., Guaita, L., Östlin, G., Hayes, M., \& Kiaeerad, F. 2015, A\&A, 580, A91

Sandin, C., Becker, T., Roth, M. M., et al. 2010, A\&A, 515, A35

Sandin, C., Weilbacher, P., Streicher, O., Walcher, C. J., \& Roth, M. M. 2011

The Messenger, 144, 13 
Sandin, C., Weilbacher, P., Tabataba-Vakili, F, Kamann, S., \& Streicher, O. 2012, in Software and Cyberinfrastructure for Astronomy II, SPIE, 8451, 84510

Schaerer, D., \& Verhamme, A. 2008, A\&A, 480, 369

Schaerer, D., Hayes, M., Verhamme, A., \& Teyssier, R. 2011, A\&A, 531, A12

Shapley, A. E., Steidel, C. C., Pettini, M., \& Adelberger, K. L. 2003, ApJ, 588, 65

Shimasaku, K., Kashikawa, N., Doi, M., et al. 2006, PASJ, 58, 313

Shioya, Y., Taniguchi, Y., Sasaki, S. S., et al. 2009, ApJ, 696, 546

Sobral, D., Matthee, J., Darvish, B., et al. 2015, ApJ, 808, 139

Song, M., Finkelstein, S. L., Gebhardt, K., et al. 2014, ApJ, 791, 3

Taniguchi, Y., Shioya, Y., Ajiki, M., et al. 2003, J. Kor. Astron. Soc., 36, 123

Tapken, C., Appenzeller, I., Mehlert, D., Noll, S., \& Richling, S. 2004, A\&A 416, L1

Tapken, C., Appenzeller, I., Gabasch, A., et al. 2006, A\&A, 455, 145

Tapken, C., Appenzeller, I., Noll, S., et al. 2007, A\&A, 467, 63

Terlevich, R., \& Melnick, J. 1981, MNRAS, 195, 839

Teyssier, R., Chapon, D., \& Bournaud, F. 2010, ApJ, 720, L149
Turner, J. E. 2010, in Canary Islands Winter School of Astrophysics, Vol. XVII 3D Spectroscopy in Astronomy, eds. E. Mediavilla, S. Arribas, M. Roth,

J. Cepa-Nogue, \& F. Sanchez (Cambridge University Press), 87 van Dokkum, P. G. 2001, PASP, 113, 1420

Verhamme, A., Schaerer, D., \& Maselli, A. 2006, A\&A, 460, 397

Verhamme, A., Schaerer, D., Atek, H., \& Tapken, C. 2008, A\&A, 491, 89

Verhamme, A., Dubois, Y., Blaizot, J., et al. 2012, A\&A, 546, A111

Wall, J. V. 1996, QJRAS, 37, 519

Wisnioski, E., Förster Schreiber, N. M., Wuyts, S., et al. 2015, ApJ, 799, 209

Wisotzki, L., Bacon, R., Blaizot, J., et al. 2015, A\&A, in press, DOI: 10.1051/0004-6361/201527384

Wold, I. G. B., Barger, A. J., \& Cowie, L. L. 2014, ApJ, 783, 119

Yamada, T., Matsuda, Y., Kousai, K., et al. 2012, ApJ, 751, 29

Yang, H., Malhotra, S., Gronke, M., et al. 2015, ApJ, submitted [arXiv: 1506.02885]

Zheng, Z., \& Wallace, J. 2014, ApJ, 794, 116

Zitrin, A., Labbé, I., Belli, S., et al. 2015, ApJ, 810, L12 


\section{Appendix A: Notes on individual objects}

In the following, we detail the observed $\mathrm{H} \alpha$ velocity fields for each galaxy. We compare our maps to the photometric Ly $\alpha$ properties derived from the HST images from Paper II and H I observations from Paper III (see Sect. 4).

\section{A.1. LARS 1 (Mrk 259)}

With $L_{\mathrm{Ly} \alpha}=8 \times 10^{41} \mathrm{erg} \mathrm{s}^{-1}$ and $E W_{\mathrm{Ly} \alpha}=33 \AA$ LARS 1 is a strong Ly $\alpha$ emitting galaxy. A detailed description of the photometric properties of this galaxy was presented in Paper I. If LARS 1 were at high- $z$, it would easily be selected in conventional narrowband imaging surveys (Paper IV). The galaxy shows a highly irregular morphology. Its main feature is UV bright complex in the north-east that harbours the youngest stellar population. As already noted in Paper II a filamentary structure emanating from the north-eastern knot is seen in $\mathrm{H} \alpha$. Towards the south-east, numerous smaller star-forming complexes are found that blend in with an older stellar population.

In contrast with the irregular appearance, the line-of-sight velocity field of LARS 1 is rather symmetric. The velocity field is consistent with a rotating galaxy. The kinematical centre is close to centre of the PMAS FoV and the kinematical axis appears to run from the north-west to the south-east. We measure $v_{\text {shear }}=56 \pm 1 \mathrm{~km} \mathrm{~s}^{-1}$. The GBT single dish H I spectrum of this source is reminiscent of a classic double-horn profile, also an obvious sign of rotation, with a peak separation consistent with our $v_{\text {shear }}$ measurement.

The highest velocity dispersions $\left(v_{\mathrm{FWHM}} \approx 150 \mathrm{~km} \mathrm{~s}^{-1}\right)$ are found in the north-eastern region. Here LARS 1 also shines strong in $\operatorname{Ly} \alpha$, but while a fraction of $\operatorname{Ly} \alpha$ appears to escape directly towards us, a more extended Ly $\alpha$ halo around this part is indicative of resonant scatterings in the circum-galactic gas (see also Paper I). In constrast, in the south-western part of the galaxy, where we observe the lowest velocity dispersions $\left(v_{\mathrm{FWHM}} \approx 70 \mathrm{~km} \mathrm{~s}^{-1}\right)$, Ly $\alpha$ photons do not escape along the line of sight.

\section{A.2. LARS 2 (Shoc 240)}

Anywhere in LARS 2 where $\mathrm{H} \alpha$ photons are produced, Ly $\alpha$ photons emerge along the line of sight. LARS 2 is the galaxy with the highest global escape fraction of Ly $\alpha$ photons $\left(f_{\mathrm{esc}}^{\mathrm{Ly} \alpha}=\right.$ $52.1 \%)$ and the highest $\mathrm{Ly} \alpha / \mathrm{H} \alpha$ ratio $(\mathrm{Ly} \alpha / \mathrm{H} \alpha=4.53)$.

With $v_{\text {shear }}=23 \pm 2 \mathrm{~km} \mathrm{~s}^{-1}$, LARS 2 shows the smallest $v_{\text {shear }}$ in our sample. However, we consider this value as a lower limit since we missed in our pointing the southernmost star-forming knot. Our observed velocity field appears to be disturbed, but we could envision a kinematical axis orthogonal to the photometric major axis, i.e. roughly from west to east. This idea is supported by our VLA imaging of this source (see Fig. 7 in Paper III). The H I velocity field also indicates $v_{\text {shear }} \approx 30 \mathrm{~km} \mathrm{~s}^{-1}$, meaning that our incomplete measurement gives a sensible lower limit. Within the observed region, our velocity dispersion map is rather uniform, with $v_{\text {VFWHM }} \approx 100 \mathrm{~km} \mathrm{~s}^{-1}$.

\section{A.3. LARS 3 (Arp 238)}

LARS 3 is the south-eastern nucleus of the violently interacting pair of similar sized spiral galaxies Arp 238. With a global $f_{\mathrm{esc}}^{\mathrm{Ly} \alpha}=0.1$ and $L_{\mathrm{Ly} \alpha}=10^{41} \mathrm{erg} \mathrm{s}^{-1}$, this dust-rich, luminous infrared galaxy is a relatively weak Ly $\alpha$ emitter. Imaging by
VLA HI reveals an extended tail towards the west. This tidal tail is much larger than the optical dimensions of the pair Arp 238 (see also Cannon et al. 2004, for similar extended tidal H I structures around the Ly $\alpha$ emitting star bursts Tol 1924-416 and IRAS08339+6517).

In $\mathrm{H} \alpha$ the main kinematical axis runs from west to east and the radial velocity field appears symmetric, although slightly disturbed towards the north and the south. We measure $v_{\text {shear }} \approx$ $140 \mathrm{~km} \mathrm{~s}^{-1}$, a value that is in the domain of typical maximum velocities of inclination corrected $\mathrm{H} \alpha$ rotation curves of spiral galaxies (e.g. Epinat et al. 2008a, 2010; Erroz-Ferrer et al. 2015). With $v_{\text {FWHM }} \gtrsim 300 \mathrm{~km} \mathrm{~s}^{-1}$ highest velocity dispersions are observed in the western part. The largest Ly $\alpha$ surface brightness is also observed in the western part of the nucleus. Ly $\alpha$ appears in absorption in the eastern region, where we also see a minimum in velocity dispersions with $v_{\mathrm{FWHM}} \approx 70 \mathrm{~km} \mathrm{~s}^{-1}$.

\section{A.4. LARS 4 (SDSS J130728.45+542652.3)}

Although LARS 4 is similar to LARS 1 in terms of dust content and star formation rate, globally this galaxy shows Ly $\alpha$ in absorption. The galaxy can be photometrically decomposed into two main components: an elongated lower surface brightness structure in the west and a more puffed up and luminous companion in the east. The eastern structure is tilted at $\approx 40^{\circ}$ with respect to the western one.

Compared to the highly irregular morphology in UV and $\mathrm{H} \alpha$, the radial velocity field appears rather regular, with a moderate amplitude of $v_{\text {shear }} \approx 75 \mathrm{~km} \mathrm{~s}^{-1}$ along a well-defined axis from west to east. The kinematic centre appears to be right between the two photometric components, indicating that the observed shearing is the velocity difference between the merging components and not a consequence of rotation. The lack of a welldefined simple and organised disk is further supported by the absence of a double-horn profile in the GBT single-dish H I profile. Nevertheless, higher sensitivity VLA imaging results show a coherent velocity field on larger scales with an amplitude comparable to $v_{\text {shear }}$ (Paper III, Fig. 9).

In the dispersion map, the two components are clearly distinguishable, with the eastern component showing higher velocity dispersions $\left(v_{\mathrm{FWHM}} \approx 100 \mathrm{~km} \mathrm{~s}^{-1}\right)$ than the western $\left(v_{\mathrm{FWHM}} \approx 65 \mathrm{~km} \mathrm{~s}^{-1}\right)$. In both regions, however, Ly $\alpha$ is seen in absorption. The highest velocity dispersions are observed at the boundary region where the two components are separated photometrically, but here only a little Ly $\alpha$ is escaping.

\section{A.5. LARS 5 (Mrk 1486)}

In the HST images LARS 5 appears as a small (i.e. projected diameter $d \approx 5 \mathrm{kpc}$ ) highly inclined edge-on disk. Within the apparent disk Ly $\alpha$ appears in absorption but the galaxy shows an extended halo, which is azimuthally symmetric at low surface brightness isophotes, while brighter isophotes resemble more the elongated $\mathrm{H} \alpha$ shape. Most prominent in the $\mathrm{H} \alpha$ image are filamentary finger-like structures extending below and above the disk, reminiscent of an outflowing wind.

Although the profile of our single-dish H I observations shows multiple peaks close to the noise level that might correspond to the peaks of a faint double-horn profile (Paper III), our $\mathrm{H} \alpha$ kinematics appear incompatible with a typical disk scenario: Firstly, our measured $v_{\text {shear }}=37 \mathrm{~km} \mathrm{~s}^{-1}$ would indicate a rotation curve with a very low amplitude, which is untypical even for a small disk (e.g. in the largest $\mathrm{H} \alpha$ disk sample of 
Epinat et al. 2010, for $d \leq 5 \mathrm{kpc}$ the maximum rotation curve velocity is on average $95 \mathrm{~km} \mathrm{~s}^{-1}$ ). Secondly, there is apparent asymmetry in the spatial distribution of the $v_{\text {LOS }}$ values, i.e. a large sector in the south-west is characterised by similar line-of-sight velocities $\left(v_{\mathrm{LOS}} \sim+20 \mathrm{~km} \mathrm{~s}^{-1}\right)$, while only a small sector in the north-east shows blueshifted $\mathrm{H} \alpha$ emission $\left(v_{\mathrm{LOS}} \sim-40 \mathrm{~km} \mathrm{~s}^{-1}\right)$.

The most prominent feature in the velocity dispersion map is a biconical zone of increasing velocity dispersions (from $\sim 120 \mathrm{~km} \mathrm{~s}^{-1}$ to $\sim 220 \mathrm{~km} \mathrm{~s}^{-1}$ ) with increasing distance from the centre. The base of this zone coincides the brightest region in $\mathrm{UV}$ and $\mathrm{H} \alpha$ in the south-western sector. We emphasise that the seeing PSF FWHM for this observation is $1.3^{\prime \prime}$, thus $\sim 2 \times$ the extent of the $0.5^{\prime \prime} \times 0.5^{\prime \prime}$ spaxels used on LARS 5. However, as the kinematical centre is not co-spatial with the base of the cones, and since the velocity gradient around the base of the cone is very weak we are confident that this feature is real and not caused by PSF smearing effects.

\section{A.6. LARS 6 (KISSR 2019)}

With $\lesssim 1 M_{\odot} \mathrm{yr}^{-1}$ conversion of gas into stars, LARS 6 shows the lowest SFR in the sample (Paper II). The main star-forming knot is in the north with a tail of much smaller and fewer luminous knots extending to the south. In our seeing limited data cubes we cannot disentangle these individual knots photometrically. Ly $\alpha$ is seen in absorption, even on the smallest scales.

Shearing is observed between the main component and the tail, although the amplitude is moderate $\left(v_{\text {shear }}=52 \mathrm{~km} \mathrm{~s}^{-1}\right)$. The main component also shows higher velocity dispersions $\left(v_{\mathrm{VFWHM}} \approx 70 \mathrm{~km} \mathrm{~s}^{-1}\right)$ than the tail $\left(v_{\mathrm{VFWHM}} \approx 50 \mathrm{~km} \mathrm{~s}^{-1}\right)$. Overall this galaxy has the lowest observed velocity dispersion in the sample.

Our GBT single-dish H I measurements are severely contaminated by the nearby field spiral UGC 10028. However, newly obtained but as of yet unpublished VLA D-configuration images allow a first-order separation from LARS 6 and this companion. From these data, coherent rotation is present in the H I gas associated with LARS 6 at a level roughly consistent with the $v_{\text {shear }}$ estimate we obtain from PMAS.

\section{A.7. LARS 7 (IRAS F13136+2938)}

In the continuum LARS 7 resembles a highly inclined disk comparable to LARS 5, but in $\mathrm{H} \alpha$ an almost azimuthally symmetric structure emerges that is highly distended with respect to the elongated continuum morphology. Moreover, two extended low surface brightness red lobes are found at the opposite ends of the inclined disk, reminiscent of a shell-like structure. This is suggestive of a recent merger event (e.g. Bettoni et al. 2011). In Ly $\alpha$ images LARS 7 appears even more extended, with the bright isophotes following the $\mathrm{H} \alpha$ shape and low surface brightness isophotes resembling a more scaled up version of the apparent disk.

For similar reasons as outlined for LARS 5 in Sect. A.5, our $\mathrm{H} \alpha$ kinematics argue against a typical disk scenario: we observe a low shearing amplitude; with $v_{\text {shear }}=31 \mathrm{~km} \mathrm{~s}^{-1}$ even lower than in LARS 5. Moreover, the line of sight velocity maps appears disturbed compared to that expected for a classical disk. Our GBT single dish H I observations reveal a single broad $\left(92 \mathrm{~km} \mathrm{~s}^{-1}\right)$ line. LARS 7 is the only object where the GBT linewidth is incompatible to its integrated $\mathrm{H} \alpha$ linewidth (cf. Sect. 6.2). A main kinematical axis can be envisioned to run from north-east to south-west along the continuum major axis, but in the north-west and south-east the $v_{\text {LOS }}$ values do not follow this weak gradient at all. Overall the galaxy shows high velocity dispersion in $\mathrm{H} \alpha\left(v_{\mathrm{FWHM}} \gtrsim 160 \mathrm{~km} \mathrm{~s}^{-1}\right)$, with the lowest values occurring in north-east $\left(v_{\mathrm{FWHM}} \approx 120 \mathrm{~km} \mathrm{~s}^{-1}\right)$, where the brightest fluxes in $\mathrm{Ly} \alpha$ and the highest $\mathrm{Ly} \alpha / \mathrm{H} \alpha$ ratios are observed.

\section{A.8. LARS 8 (SDSS-J125013.50+073441.5)}

LARS 8 appears as a face-on disk with the highest metalicity of the sample. The apparent disk also possesses a highly dust obscured nucleus. Nevertheless, there is a high degree of irregularity compared to classical disks and spiral arms are not well defined. As traces of shell-like structures are visible in the inner parts and in the outskirts of the galaxy (cf. Fig. 6 in Paper I), LARS 8 could be classified as a shell system hinting at a recent merger event (e.g. Bettoni et al. 2011). The Ly $\alpha$ light distribution of LARS 8 does not resemble that seen in $\mathrm{H} \alpha$. The brightest Ly $\alpha$ zone is in the north of the disk, while in the south Ly $\alpha$ appears only in absorption. At lower surface brightness isophotes a Ly $\alpha$ halo becomes visible. With an equivalent width $E W_{\mathrm{Ly} \alpha}=20.3 \AA$ this galaxy would be selected as LAE in conventional narrowband surveys.

Our $v_{\text {LOS }}$ map seems disk-like. Assuming an infinitely thin disk the ellipticity of 0.2 (Paper IV) implies an inclination of $40^{\circ}$, hence, our observed $v_{\text {shear }}=155 \mathrm{~km} \mathrm{~s}^{-1}$ translates to $v_{\max }=$ $241 \mathrm{~km} \mathrm{~s}^{-1}$. The GBT single-dish H I profile shows a broad, possibly double-peaked profile. Although these peaks are not significantly separated from the overall H I signal, the implied velocity difference of $\sim 300 \mathrm{~km} \mathrm{~s}^{-1}$ appears consistent with our $v_{\text {shear }}$ measurement. The orientation of the velocity field from VLA interferometric $\mathrm{HI}$ observations is qualitatively consistent with our PMAS $\mathrm{H} \alpha$ observations, although at the VLA D-configuration beam size of $72^{\prime \prime}$ LARS 8 appears only marginally resolved.

Elevated velocity dispersions with $v_{\mathrm{FWHM}} \gtrsim 160 \mathrm{~km} \mathrm{~s}^{-1}$ are apparent to extend orthogonally to the kinematical axis along the $0 \mathrm{~km} \mathrm{~s}^{-1}$ iso $v_{\mathrm{LOS}}$ contour. In the other regions, the $v_{\mathrm{FWHM}}$ map is rather flat, typically with $v_{\text {FWHM }} \sim 100 \mathrm{~km} \mathrm{~s}^{-1}-$ a value that is also still commonly observed within the sample of 153 local spirals by Epinat et al. (2010). Given the strong gradient in the velocity field, the observed elevated velocity dispersions are likely a result of PSF smearing effects.

\section{A.9. LARS 9 (IRAS 08208+2816)}

This luminous infrared galaxy has highly irregular morphology. Numerous star-forming knots are visible in $\mathrm{H} \alpha$ along two arms of large extend that are connected to a central, very bright $\mathrm{H} \alpha$ nucleus. At the end of the southern tail, a foreground star appears in projection; contributions from this star have been masked out in the PMAS data. In LARS 9 Ly $\alpha$ is absorbed almost everywhere along the line of sight towards the star-forming regions. Nevertheless, this galaxy is embedded in a faint extended Ly $\alpha$ fuzz. Morphologically, this fuzz broadly traces a scaled up version of its optical and $\mathrm{H} \alpha$ shape. Having a globally integrated Ly $\alpha$ luminosity of $L_{\mathrm{Ly} \alpha} \approx 3 \times 10^{41} \mathrm{erg} \mathrm{s}^{-1}$ and an equivalent width of $E W_{\mathrm{Ly} \alpha} \approx 8 \AA$, this galaxy would not be selected by its Ly $\alpha$ emission at high- $z$ in conventional narrowband surveys.

Since LARS 9 extends more than $\sim 0.5^{\prime}$ on the sky, we needed to cover it with two PMAS $16^{\prime \prime} \times 16^{\prime \prime}$ pointings (Fig. 6). The line-of-sight velocity field for this galaxy is very peculiar. From the north to the centre, a weak $\left(\sim 100 \mathrm{~km} \mathrm{~s}^{-1}\right)$ gradient from blueshifted to systemic velocity is apparent. From the centre 

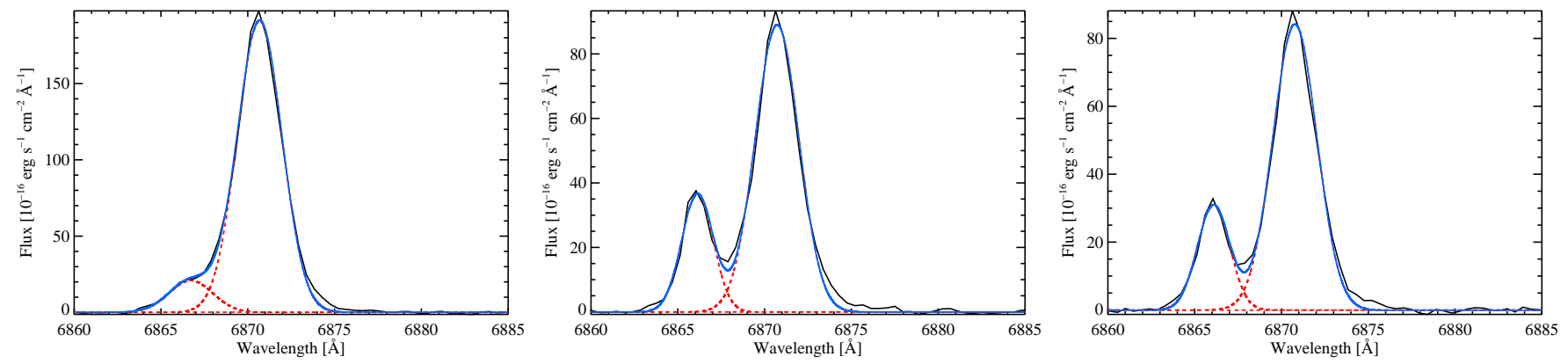

Fig. A.1. Representative LARS $9 \mathrm{H} \alpha$ line profiles in blue hatched region of Fig. 6 described by a fit using two Gaussian components. The red dashed lines show the individual components and the blue line shows the sum, while the black line shows the profile as observed. For all profiles shown the single component fit used to create the map shown in Fig. 6 converged on the stronger red component.
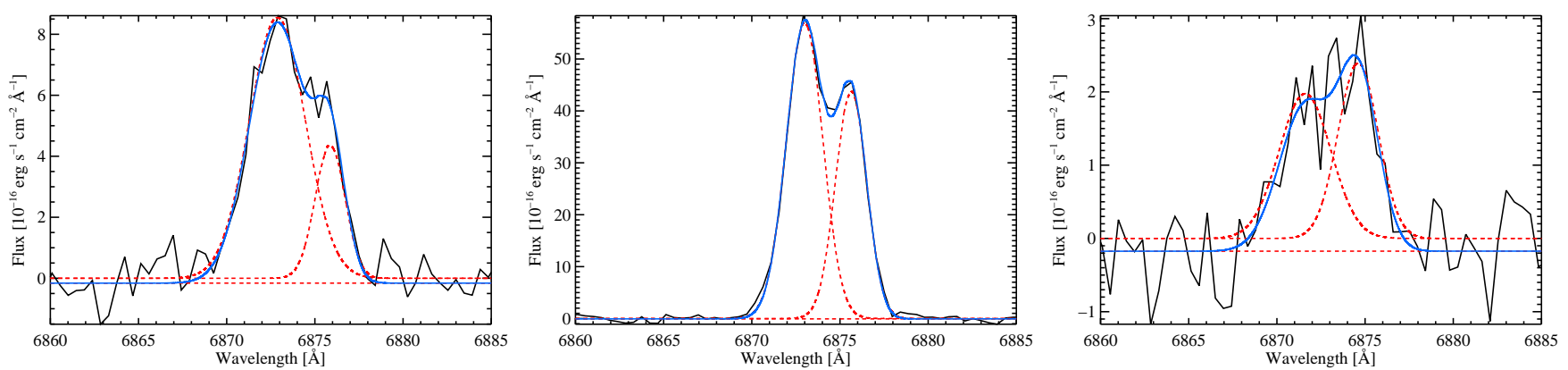

Fig. A.2. Representative LARS $9 \mathrm{H} \alpha$ line profiles in red hatched region of Fig. 6, similar to Fig. A.1. The single component fit used to create the map shown in Fig. 6 converged on the stronger blue component in the centre panel, but for the profiles shown in the left and right panels the single component fit is artificially broadened.
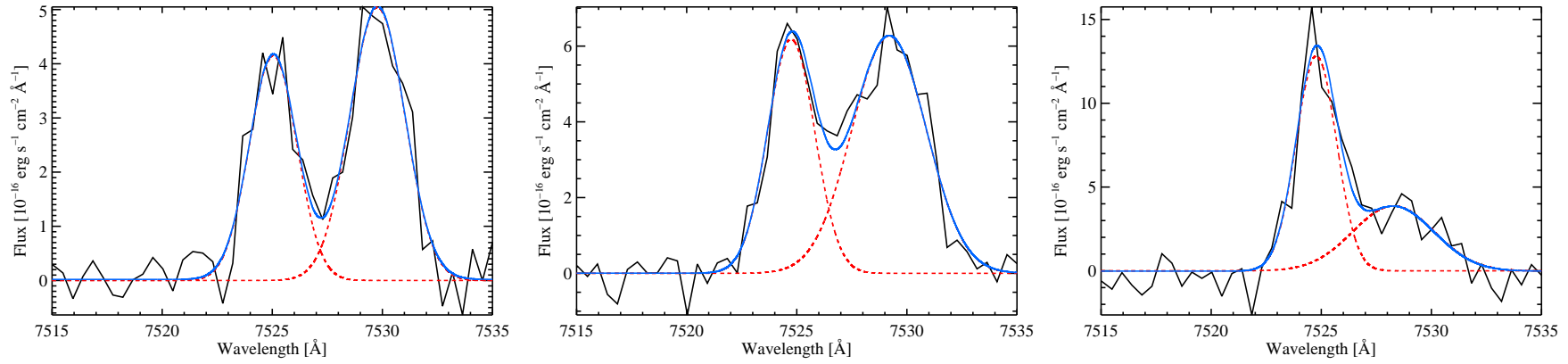

Fig. A.3. Representative LARS $13 \mathrm{H} \alpha$ line profiles in blue hatched region of Fig. 7, similar to Fig. A.1. The single component fit used to create the map shown in Fig. 7 converged on the stronger blue component in the right panel, but for the profiles shown in the left and centre panels the single component fit is artificially broadened.

this weak gradient continues towards the south. The southern tail then shows an opposite gradient from red- to blueshifts. In this south- and south-western zone, a single Gaussian often is not an optimal representation of the observed $\mathrm{H} \alpha$ lines. In this zone the spectral profile is sometimes asymmetric with an extended red or blue wing, but often it also shows clearly doublepeaked morphology (for examples see Fig. A.1). In some of these regions, our fit tries to capture both lines, hence, the obtained line-of-sight velocity is centred between the peaks and the linewidth appears very broad (e.g. left and right panels of Fig. A.2). In some other spaxels, when the secondary peak is very weak or when only an extended wing is seen, the fit converges to the stronger line. The affected zones have hatched rectangles overlaid in Fig. 6. These zones harbour a kinematical distinct secondary component. This secondary component does not follow the large-scale motions of the northern component, to which the nucleus also belongs. We indicate, with red and blue hatchings in Fig. 6, the regions of this component that are redand blueshifted with respect to the systemic velocity (as given by the nucleus). As a natural outcome from this qualitative analysis the emission in south-eastern tail of LARS 9 is solely coming from this secondary component. Also, a significant fraction of Ly $\alpha$ photons appear to escape towards the observer only at the end of this tail.

From our PMAS observation we conclude that LARS 9 is a closely interacting pair of galaxies in an advanced stage of merging. The interaction scenario is also supported by our $21 \mathrm{~cm}$ observations. The integrated GBT spectrum shows a 
broad single line, indicating that the bulk of the $\mathrm{H}$ i is not partaking in an ordered flat rotation. LARS 9 was only marginally resolved in our VLA HI maps (Paper III). However, as of yet unpublished VLA C configuration observations (K. Fitzgibbon, in prep.) show extended HI towards the west, enclosing the galaxy SDSS J082353.65+280622.2. For this galaxy, no spectroscopic redshift is available, but the photometric redshift agrees with being associated to LARS 9. These observations therefore strongly suggest that a third system is significantly involved in the interaction. Moreover, the VLA C-configuration observations of LARS 9 show a peak in the second-moment maps that trace the random motions of $\mathrm{H}$ I that coincides spatially with the zones of multiple H II components in our PMAS maps. Hence, at these locations (hatched regions in Fig. 6) Ly $\alpha$ photons of both distinct kinematical components are likely scattered by $\mathrm{H} \mathrm{I}$ at resonance.

\section{A.10. LARS 10 (Mrk 0061)}

Similar to LARS 1 this galaxy appears morphologically to be in an advanced merger state. It possesses a large UV bright core in the north-west and a spur of smaller, less luminous starforming regions towards the south-east. Several redder low surface brightness structures are seen outside the main body of the galaxy, reminiscent of the appearance of shell galaxies (e.g. Marino et al. 2009; Bettoni et al. 2011). From the central starforming parts $\operatorname{Ly} \alpha$ is only seen in absorption, while a faint halo emerges at larger radii. When considering solely the central parts the galaxy would remain undetected in high- $z$ LAE surveys since $E W_{\mathrm{Ly} \alpha}=8 \AA$. However, when integrating over the lower surface brightness emission $E W_{\mathrm{Ly} \alpha}$ rises to $31 \AA$ and with its luminosity of $L_{\mathrm{Ly} \alpha}=2 \times 10^{41} \mathrm{erg} \mathrm{s}^{-1}$ the galaxy would be within the sensitivity of the deepest contemporary LAE surveys (Rauch et al. 2008; Bacon et al. 2015).

The line-of-sight velocity field is symmetric and consistent with rotation and contrasts the irregular continuum morphology. The velocity gradient running from the south-east to the northwest is weak and we measure $v_{\text {shear }}=36 \mathrm{~km} \mathrm{~s}^{-1}$. The seeing for this observation was very substantial $\left(1.5^{\prime \prime}\right.$ FWHM, i.e $3 \times$ the size of the $0.5^{\prime \prime} \times 0.5^{\prime \prime}$ spaxels). Despite the weak gradient, this leads to non-negligible PSF smearing effects in the dispersion map. Moreover, because of the short exposure time and the rather low $\mathrm{H} \alpha$ flux, this galaxy has the lowest $\mathrm{S} / \mathrm{N}$ per spaxel of the whole sample; this manifests in a noisy $v_{\text {FWHM }}$ map. Both of these effects essentially make local disturbances in the velocity dispersion untraceable in our LARS 10 data set, but our flux weighted global measurement of $\sigma_{0} \approx 40 \mathrm{~km} \mathrm{~s}^{-1}$ is robust against these nuisances.

The GBT integrated H I spectrum shows a broad single line profile ( $\left.280 \mathrm{~km} \mathrm{~s}^{-1} \mathrm{FWHM}\right)$, however, at low signal to noise. This appears hard to reconcile with the $\mathrm{H} \alpha$ results. If real, it might indicate that a large fraction of neutral gas in and around LARS 10 is kinematically in a different state than the gas around the galaxy's star-forming regions.

\section{A.11. LARS 11 (SDSS J140347.22+062812.1)}

This galaxy appears as a highly inclined edge-on disk in the continuum and UV that is slightly thicker when seen in $\mathrm{H} \alpha$. According to the $\mathrm{H} \alpha$ and UV emission, stronger star formation occurs in the south-eastern zone of the projected disk. In Ly $\alpha$ a mildly extended halo above and below the plane is visible, with the isophotal contours approximately preserving the axis ratio of $\mathrm{H} \alpha$. Within the disk Ly $\alpha$ occurs exclusively in absorption.

Running along the disk from the north-west to the southeast a strong gradient in the $v_{\text {LOS }}$ map is apparent. The observed shearing amplitude $v_{\text {shear }}=150 \mathrm{~km} \mathrm{~s}^{-1}$ is consistent with this gradient being caused by rotation (e.g. Epinat et al. 2008a, 2010; Erroz-Ferrer et al. 2015). Unfortunately the beam of our GBT single-dish H I observations is likely contaminated by other sources. Nevertheless, within the multiple peaks in the GBT spectrum a double-horn profile at a velocity separation consistent with our $\mathrm{H} \alpha v_{\text {shear }}$ measurement is visible. Although the projected height of the disk is similar to the PSF FWHM, the dispersion map shows traces of higher velocity dispersions above and below the disk $\left(v_{\mathrm{VFWHM}} \approx 200 \mathrm{~km} \mathrm{~s}^{-1}\right)$ than within $\left(v_{\mathrm{VFWHM}} \approx 150 \mathrm{~km} \mathrm{~s}^{-1}\right)$, especially around the star-forming regions in the south-east. However, the elevated dispersions near the kinematic centre are caused by PSF smearing of the strong gradient in the $v_{\text {LOS }}$ field.

\section{A.12. LARS 12 (LEDA 27453)}

Because of its modest $E W_{\mathrm{Ly} \alpha}=13 \AA$, this UV bright merger would not be selected as a LAE at high-z. At its brightest knot in UV and $\mathrm{H} \alpha$ the galaxy shows $\operatorname{Ly} \alpha$ only in absorption. Ly $\alpha$ only appears in emission at larger radii, where a number of fainter star-forming regions can be appreciated. Moreover, LARS 12 is embedded in a faint, low surface brightness Ly $\alpha$ halo.

The non-regular $\mathrm{H} \alpha$ velocity field indicates complex kinematics. In the north-western corner a strong $\sim 140 \mathrm{~km} \mathrm{~s}^{-1}$ gradient over $\sim 1.5^{\prime \prime}\left(\sim 2.8 \mathrm{kpc}\right.$ at $\left.d_{\text {LARS12 }}=470.5 \mathrm{Mpc}\right)$ is observed, but the velocity field is essentially flat from there towards the south-west. The high velocity and velocity dispersion in the values seen in the south are not significant, as the low $\mathrm{S} / \mathrm{N}$ of this broad line in these binned spaxels lead to a $50 \%$ error on the determined $v_{\text {LOS }}$ and $\sigma_{0}$ value. Because of PSF smearing effects the strong velocity gradient is responsible for the region of elevated velocity dispersions in the north-western corner.

For LARS 12 adaptive optics Paschen $\alpha$ IFS observations have been presented in Gonçalves et al. (2010); object ScUVLG 093813 in their nomenclature. Their line-of-sight velocity field is qualitatively fully consistent with ours, but their higher resolution data allows them to pinpoint the highest redshifted values directly to the two filaments that extend towards the north-west. Since they are not affected by PSF smearing their dispersion map is not contaminated by high-velocity dispersions in the northwest. Of course, our artifact in the south is also absent from their map. Nevertheless, their $\sigma_{0}$ value of $62 \mathrm{~km} \mathrm{~s}^{-1}$ is in good agreement with our measurement of $72 \mathrm{~km} \mathrm{~s}^{-1}$.

\section{A.13. LARS 13 (IRAS 01477+1254)}

Based on its highly irregular morphology this starburst system is clearly interacting. As LARS 13 shows a narrow Ly $\alpha$ equivalent width $\left(E W_{\text {Ly } \alpha}=6 \AA\right)$, it would not be selected as a LAE at high redshift in conventional narrowband surveys. Overall, only $1 \%$ of $\operatorname{Ly} \alpha$ photons are escaping and the resulting Ly $\alpha$ luminosity is $L_{\text {Ly } \alpha}=7 \times 10^{41} \mathrm{erg} \mathrm{s}^{-1}$.

The complex $v_{\text {LOS }}$ map of this galaxy is characterised by a gradient from blue- to redshifts along the east-western axis for the northern component, as well as an gradient from blue-to redshifts from south to north for the eastern component. In the north-western zone where the longitudinally aligned structure 
overlaps with the latitudinally aligned one, our single component Gaussian is not an optimal representation of the observed $\mathrm{H} \alpha$ line profiles. In this region, the profile is often asymmetric with an extended blue wing (e.g. right panel of Fig. A.3). Some spaxels show a double-peaked $\mathrm{H} \alpha$ profile (e.g. left and centre panels of Fig. A.3). The affected zone has a hatched rectangle overlaid in Fig. 7. In the region where two equally strong peaks are prominent in the spectrum, our fit tries to fit a broad line that encloses both lines; these spaxels can be seen in the east running as a diagonal line of broad velocity dispersions from north-west to south-east. Spaxels east of this demarcation show an extended blue wing. We interpret this to mean that we are seeing separate components of ionised gas kinematics, where one component belongs to the latitudinal elongated structure while the other belongs to the longitudinal elongated structure. The spatial and spectral proximity of both regions indicates that the galaxy is still in an ongoing interaction with both progenitors not having fully coalesced.

For LARS 13 adaptive optics Paschen $\alpha$ IFS observations have been presented in Gonçalves et al. (2010); object ScUVLG 015028 in their nomenclature. However, their small FoV allows them only to sample emission from the strongest star-forming region in the western part, and so they do not have any information on the longitudinally aligned eastern part. Consequently, their observed $v_{\text {shear }}=78 \mathrm{~km} \mathrm{~s}^{-1}$ is lower than our value $\left(v_{\text {shear }}=173 \mathrm{~km} \mathrm{~s}^{-1}\right)$, since the north-eastern zone with highest $v_{\text {Los }}$ values in our map is not present in their data. In the zone where they have signal, there is good qualitative and quantitative agreement between our maps and their maps. In particular, they found high velocity dispersions $v_{\mathrm{FWHM}} \gtrsim 200 \mathrm{~km} \mathrm{~s}^{-1}$ and a weak velocity gradient in the north-western star-forming knot. In this region of high velocity dispersions $\operatorname{Ly} \alpha$ appears to escape directly towards the observer.

\section{A.14. LARS 14 (SDSS J092600.41+442736.1)}

LARS 14 is the most luminous LAE in the sample $\left(L_{\mathrm{Ly} \alpha}=\right.$ $\left.4.2 \times 10^{42} \mathrm{erg} \mathrm{s}^{-1}\right)$. It is also classified as a green pea galaxy (Cardamone et al. 2009, see also their Fig. 7). Green pea galaxies show commonly strong $\operatorname{Ly} \alpha$ emission and are also thought to be Lyman continuum leaking galaxies (Jaskot \& Oey 2014; Henry et al. 2015; Yang et al. 2015). Visible in the HST images is a small companion in the south and tidal tails extending to the east and south (Cardamone et al. 2009). Our seeing limited data does not provide enough resolution to disentangle this structure photometrically from the main component within the datacube. Kinematically, however, we observe that the southern region shears at $\approx 60-70 \mathrm{~km} \mathrm{~s}^{-1}$ with respect to the northern, with not much velocity substructure seen. In the main part we observe high velocity dispersions with $v_{\mathrm{FWHM}} \approx 175 \mathrm{~km} \mathrm{~s}^{-1}$, dropping to 80-90 $\mathrm{km} \mathrm{s}^{-1}$ towards the companion. Our modelling of the $\mathrm{H} \alpha$ profile with a simple 1D Gaussian did not capture the extended broad wings seen in the $\mathrm{H} \alpha$ profiles. These broad wings likely trace outflowing ionised gas, and are a feature regularly observed in pea galaxies (Yang et al. 2015).

For this galaxy, adaptive optics Paschen $\alpha$ IFS observations have been presented in (Gonçalves et al. and 2009, object ScUVLG 92600 in their nomenclature). They report slightly higher values, both for the shear and the dispersion. Nevertheless, our derived $v_{\text {shear }} / \sigma_{0}=0.6 \pm 0.1$ is consistent with their value of 0.51 . This ratio would be lowered by about a factor of two, if only the northern main component is considered, since its velocity offset relative to the companion dominates the shear. The main component as such therefore presents the structure with the lowest $v_{\text {shear }} / \sigma_{0}$ of our whole sample. 